\title{
TAIL MEASURE AND SPECTRAL TAIL PROCESS OF REGULARLY VARYING TIME SERIES
}

\author{
By Clément Dombry ${ }^{1}$, Enkelejd Hashorva ${ }^{2}$ And Philippe SOUlier ${ }^{3}$ \\ Université Bourgogne Franche-Comté, Université de Lausanne and \\ Université Paris Nanterre
}

\begin{abstract}
The goal of this paper is an exhaustive investigation of the link between the tail measure of a regularly varying time series and its spectral tail process, independently introduced in [Owada and Samorodnitsky (2012)] and [Stochastic Process. Appl. 119 (2009) 1055-1080]. Our main result is to prove in an abstract framework that there is a one-to-one correspondence between these two objects, and given one of them to show that it is always possible to build a time series of which it will be the tail measure or the spectral tail process. For nonnegative time series, we recover results explicitly or implicitly known in the theory of max-stable processes.
\end{abstract}

1. Introduction. Regular variation is a fundamental concept for the extreme value analysis of time series; see, for instance, Kulik (2016) and the articles in this collection for a recent overview. For stationary multivariate time series, Basrak and Segers (2009) proved that regular variation is equivalent to the existence of the so-called tail and spectral tail processes which capture the entire tail behaviour of the series. An important property of the spectral tail process is the time change formula also proved by Basrak and Segers (2009). Recently, Segers, Zhao and Meinguet (2017) and Owada and Samorodnitsky (2012) introduced the tail measure of a regularly varying, but not necessarily stationary, time series. The tail measure is a homogeneous measure on the sequence space and it is shift-invariant for a stationary time series. This is an advantage with respect to the tail process which is never stationary. In addition, the tail process can be recovered from the tail measure and it appears that the time change formula is a straightforward consequence of the shift invariance of the tail measure.

A very natural question arises: given the tail process or the spectral tail process of a time series, is it possible to reconstruct explicitly the tail measure? Furthermore, since the tail and spectral tail processes can be defined solely in terms of the tail measure, given a process satisfying the time change formula, is it possible

Received October 2017; revised April 2018.

${ }^{1}$ Supported in part by the Bourgogne Franche-Comté region (Grant OPE-2017-0068).

${ }^{2}$ Supported in part by SNSF Grant no. 200021-175752/1.

${ }^{3}$ Supported in part by LABEX MME-DII.

MSC2010 subject classifications. 60G70.

Key words and phrases. Regularly varying time series, tail measure, spectral tail process, time change formula. 
to define a tail measure and a time series of which it is the spectral tail process? The latter question was recently solved positively by Janßen (2017) who shows that given a process satisfying the time change formula, there exists a time series of which it is the spectral tail process.

The purpose of this paper is twofold. In Section 2, we will attempt to present a systematic theory of tail measures on a abstract complete separable metric space and their representations, with a particular focus on shift-invariant tail measures. This is done by means only of measure theory and the homogeneity and shift invariance properties of a tail measure, without any appeal to regular variation or probabilistic asymptotic arguments. We establish in Theorem 2.4 the stochastic representation of tail measures with a characterization of the shift invariance. These stochastic representations have a property similar to the time change formula which we refer to as the tilt shift formula. The spectral tail process associated to the tail measure is then related to its stochastic representation and we prove that there is a one-to-one correspondence between spectral tail processes, stochastic representations and shift invariant tail measures in Theorem 2.9.

In Section 2.5, we discuss dissipative representations of tail measures and characterize the existence of such representations, which are deeply related to the mixed moving average representation of max-stable processes. We conclude this general investigation of tail measure by introducing maximal indices which extend the candidate extremal index of Basrak and Segers (2009).

In Section 3, the abstract tail measures introduced in Section 2 are related to be the tail measure of a regularly varying time series, in particular max-stable processes; see de Haan (1984), Davis, Klüppelberg and Steinkohl (2013) or Buhl and Klüppelberg (2016). The main result of this section is that we show that any shiftinvariant homogeneous measure $\boldsymbol{v}$ can be obtained as the tail measure of a regularly varying stationary times series. Our construction relies on a Poisson particle system, similar to the representation of max-stable sequences, and on the regular variation of Poisson point measures on abstract metric spaces. The main theoretical tool we use is the theory of $\mathcal{M}_{0}$ convergence on metric spaces and its application to regular variation, following Hult and Lindskog (2006). We also make use of the theory of convergence of random measures as set out in Kallenberg (2017). Our main result extends the above mentioned result of Janßen (2017) to our more general framework. The properties of the proposed class of stationary regularly varying time series are then studied and we show in particular that they admit extremal indices which coincide with the maximal indices introduced in Section 2.6.

\section{Tail measures on a metric space.}

2.1. Framework. The mathematical setting is the following. Let $(\mathrm{E}, \mathcal{E})$ be a measurable cone, that is, a measurable space together with a multiplication by positive scalars

$$
(u, \boldsymbol{x}) \in(0, \infty) \times \mathrm{E} \mapsto u \boldsymbol{x} \in \mathrm{E},
$$


which is measurable with respect to the product $\sigma$-field $\mathcal{B}(0, \infty) \otimes \mathcal{E} / \mathcal{E}$ and satisfies

$$
1 \boldsymbol{x}=\boldsymbol{x}, \quad u(v(\boldsymbol{x}))=(u v) \boldsymbol{x}, \quad u, v>0, \boldsymbol{x} \in \mathrm{E} .
$$

We assume that the cone admits a zero element $\mathbf{0}_{\mathrm{E}} \in \mathrm{E}$ such that $u \mathbf{0}_{\mathrm{E}}=\mathbf{0}_{\mathrm{E}}$ for all $u>0$ and that it is endowed with a pseudonorm, that is, a measurable function $\|\cdot\|_{\mathrm{E}}: \mathrm{E} \mapsto[0, \infty)$ such that $\|u \boldsymbol{x}\|_{\mathrm{E}}=u\|\boldsymbol{x}\|_{\mathrm{E}}$ for all $u>0, \boldsymbol{x} \in \mathrm{E}$ and $\|\boldsymbol{x}\|_{\mathrm{E}}=0$ implies $\boldsymbol{x}=\mathbf{0}_{\mathrm{E}}$. The triangle inequality is not required.

The space $E^{\mathbb{Z}}$ of $E$-valued sequences is endowed with the cylinder $\sigma$-algebra $\mathcal{F}=\mathcal{E}^{\otimes \mathbb{Z}}$ and a generic sequence is denoted $\boldsymbol{x}=\left(\boldsymbol{x}_{h}\right)_{h \in \mathbb{Z}}$. The sequence identically equal to $\mathbf{0}_{E}$ is denoted by $\mathbf{0}_{\mathrm{E}}$. The backshift operator $B$ on $\mathrm{E}^{\mathbb{Z}}$ is defined by $(B \boldsymbol{x})_{h}=\boldsymbol{x}_{h-1}, \boldsymbol{x} \in \mathbb{E}^{\mathbb{Z}}, h \in \mathbb{Z}$. Its iterates are denoted $B^{k}, k \in \mathbb{Z}$.

Let $H: \mathbb{E}^{\mathbb{Z}} \mapsto[0, \infty]$ be an $\mathcal{F}$-measurable function. We say that $H$ is homogeneous of order $\alpha \in \mathbb{R}$, or shortly $\alpha$-homogeneous, if $H(u \boldsymbol{x})=u^{\alpha} H(\boldsymbol{x})$ for all $u>0, x \in \mathrm{E}^{\mathbb{Z}}$.

The central object in this section is the notion of tail measure defined as follows.

Definition 2.1 (Tail measure). A tail measure with index $\alpha>0$ is a positive measure $\boldsymbol{v}$ on $\left(\mathrm{E}^{\mathbb{Z}}, \mathcal{F}\right)$ with the following properties:

(i) $\boldsymbol{v}\left(\left\{\mathbf{0}_{\mathrm{E}^{\mathbb{Z}}}\right\}\right)=0$;

(ii) $\boldsymbol{v}\left(\left\{\left\|\boldsymbol{x}_{0}\right\|_{\mathrm{E}}>1\right\}\right)=1$;

(iii) $\boldsymbol{v}\left(\left\{\left\|\boldsymbol{x}_{h}\right\|_{\mathrm{E}}>1\right\}\right)<\infty$ for all $h \in \mathbb{Z}$;

(iv) $\boldsymbol{v}$ is $\alpha$-homogeneous, that is $\boldsymbol{v}(u A)=u^{-\alpha} \boldsymbol{v}(A)$ for all $A \in \mathcal{F}$ and $u>0$.

The tail measure $v$ is called shift-invariant if furthermore

(v) $\boldsymbol{v}(B A)=\boldsymbol{v}(A)$ for all $A \in \mathcal{F}$.

The following connection of tail measures on $[0, \infty)^{\mathbb{Z}}$ and max-stable process is important.

Remark 2.2. A time series $\boldsymbol{X}=\left(\boldsymbol{X}_{h}\right)_{h \in \mathbb{Z}}$ is called $\alpha$-Fréchet max-stable if

$$
\left(n^{-1 / \alpha} \bigvee_{i=1}^{n} \boldsymbol{X}_{h}^{(i)}\right)_{h \in \mathbb{Z}} \stackrel{d}{=}\left(\boldsymbol{X}_{h}\right)_{h \in \mathbb{Z}},
$$

where $\boldsymbol{X}^{(i)}, i \geq 1$ are independent copies of $\boldsymbol{X}$. de Haan's representation theorem [de Haan (1984)] implies that any $\alpha$-Fréchet max-stable sequence $\boldsymbol{X}$ can be represented as

$$
\left(\boldsymbol{X}_{h}\right)_{h \in \mathbb{Z}} \stackrel{d}{=}\left(\bigvee_{i \geq 1} \mathbf{P}_{h}^{(i)}\right)_{h \in \mathbb{Z}},
$$

where $\sum_{i \geq 1} \delta_{\mathbf{P}^{(i)}}$ is a Poisson random measure on $[0, \infty)^{\mathbb{Z}}$ with intensity $\boldsymbol{v}$ called the exponent measure of $\boldsymbol{X}$. Provided the marginal distribution of $\boldsymbol{X}_{0}$ is standard 
$\alpha$-Fréchet, the exponent measure $\boldsymbol{v}$ is a tail measure in the sense of Definition 2.1. Conversely, for any tail measure $\boldsymbol{v}$ on $[0, \infty)^{\mathbb{Z}}$, Equation (2.1) defines an $\alpha$-Fréchet max-stable sequence with $\boldsymbol{X}_{0}$ following a standard $\alpha$-Fréchet distribution.

The following lemma is useful to characterize tail measures. According to Definition 2.1, the restriction of a tail measure $\boldsymbol{v}$ to the set $\left\{\left\|\boldsymbol{x}_{h}\right\|_{\mathrm{E}}>1\right\}$ is finite so that the Lemma allows to deal with finite measures in order to characterize $\boldsymbol{v}$.

LEMMA 2.3. Any tail measure $\boldsymbol{v}$ is $\sigma$-finite and uniquely determined by its restrictions to the sets $\left\{\left\|\boldsymbol{x}_{h}\right\|_{\mathrm{E}}>1\right\}, h \in \mathbb{Z}$.

PROOF. By property (i) of Definition 2.1, the tail measure $v$ is supported by

$$
\mathrm{E}^{\mathbb{Z}} \backslash\left\{\mathbf{0}_{\mathrm{E}^{\mathbb{Z}}}\right\}=\bigcup_{h \in \mathbb{Z}, n \geq 1} A_{h, n},
$$

with $A_{h, n}=\left\{\boldsymbol{x} \in \mathrm{E}^{\mathbb{Z}}:\left\|\boldsymbol{x}_{h}\right\|_{\mathrm{E}}>n^{-1}\right\}$. Since $\mathrm{E}^{\mathbb{Z}} \backslash\left\{\boldsymbol{0}_{\mathrm{E}} \mathbb{Z}\right\}$ is a countable union of measurable sets, we can also write it as a countable union of pairwise disjoint measurable sets. For instance, enumerating $A_{h, n}, h \in \mathbb{Z}, n \geq 1$ as $D_{i}, i \geq 1$ and taking $\mathcal{D}_{1}=D_{1}, \mathcal{D}_{i}=D_{i} \cap\left(D_{1} \cup \cdots \cup D_{i-1}\right)^{c}, i \geq 2$, we have that $\mathrm{E}^{\mathbb{Z}} \backslash\left\{\mathbf{0}_{\mathrm{E}} \mathbb{Z}\right\}=$ $\bigcup_{i \geq 1} \mathcal{D}_{i}$ with the sets $\mathcal{D}_{i}, i \geq 1$, being pairwise disjoint. Since by property (iii) and (iv), we have that $\boldsymbol{v}\left(\mathcal{D}_{i}\right)<\infty, i \geq 1$, then $\boldsymbol{v}$ is $\sigma$-finite and completely determined by its restrictions to the sets $\mathcal{D}_{i}, i \geq 1$; hence by its restriction to the sets $A_{h, n}$, $h \in \mathbb{Z}, n \geq 1$. Using further the homogeneity property (iv), it follows that $\boldsymbol{v}$ is determined by its restriction to the sets $\left\{\left\|\boldsymbol{x}_{h}\right\|_{\mathrm{E}}>1\right\}, h \in \mathbb{Z}$.

2.2. Stochastic representation of tail measures. The following theorem provides a fundamental stochastic representation of a tail measure in terms of a $E$ valued stochastic process $\boldsymbol{Z}=\left(\boldsymbol{Z}_{h}\right)_{h \in \mathbb{Z}}$ and characterizes shift-invariant tail measures.

THEOREM 2.4. A measure $\boldsymbol{v}$ on $\left(\mathrm{E}^{\mathbb{Z}}, \mathcal{F}\right)$ is a tail measure with index $\alpha>0$ if and only if there exists an $\mathrm{E}$-valued stochastic process $\boldsymbol{Z}=\left(\boldsymbol{Z}_{h}\right)_{h \in \mathbb{Z}}$ defined on a probability space $(\Omega, \mathcal{A}, \mathbb{P})$ such that

$$
\begin{array}{cl}
\mathbb{P}\left(\boldsymbol{Z}=\mathbf{0}_{\mathrm{E}}\right)=0, & \mathbb{E}\left[\left\|\boldsymbol{Z}_{0}\right\|_{\mathrm{E}}^{\alpha}\right]=1, \\
\mathbb{E}\left[\left\|\boldsymbol{Z}_{h}\right\|_{\mathrm{E}}^{\alpha}\right]<\infty & \text { for all } h \in \mathbb{Z},
\end{array}
$$

and

$$
\boldsymbol{v}(A)=\int_{0}^{\infty} \mathbb{P}(r \boldsymbol{Z} \in A) \alpha r^{-\alpha-1} \mathrm{~d} r, \quad A \in \mathcal{F} .
$$

Moreover, $\boldsymbol{v}$ is shift-invariant if and only if, for all nonnegative measurable $\alpha$ homogeneous functions $H$ and $h \in \mathbb{Z}$,

$$
\mathbb{E}\left[H\left(B^{h} \boldsymbol{Z}\right)\right]=\mathbb{E}[H(\boldsymbol{Z})]
$$


Note that in Equation (2.3), both terms may be equal to $+\infty$, for instance, if $A=$ $\left\{\|\boldsymbol{x}\|_{\mathrm{E}}>0\right\}$. This raises however no difficulty since the results from measure theory we use (e.g., Fubini-Tonneli theorem) hold true for any nonnegative functions and $\sigma$-finite measures, regardless the integrals are finite or not.

We call the identity (2.4) the tilt shift formula, abbreviated TSF. It characterizes the shift-invariance of the measure $\boldsymbol{v}$ defined by (2.3) which does not depend on the choice of a norm. It looks very much like stationarity of the process $\boldsymbol{Z}$, but let us emphasize that (2.4) is restricted to $\alpha$-homogeneous test functions so it is much weaker than stationarity. Of course, if $\boldsymbol{Z}$ is stationary then it satisfies (2.4). The tilt shift formula is equivalent to each of the following equivalent conditions which will also be referred to indifferently as the TSF:

(i) for all nonnegative measurable 0-homogeneous functions $H_{0}: \mathrm{E}^{\mathbb{Z}} \rightarrow$ $[0, \infty]$ and $h \in \mathbb{Z}$,

$$
\mathbb{E}\left[\left\|\boldsymbol{Z}_{0}\right\|_{\mathrm{E}}^{\alpha} H_{0}\left(B^{h} \boldsymbol{Z}\right)\right]=\mathbb{E}\left[\left\|\boldsymbol{Z}_{h}\right\|_{\mathrm{E}}^{\alpha} H_{0}(\boldsymbol{Z})\right]
$$

(ii) for all nonnegative measurable functions $K: \mathbb{E}^{\mathbb{Z}} \rightarrow \mathbb{R}$ and $h \in \mathbb{Z}$,

$$
\mathbb{E}\left[\left\|\boldsymbol{Z}_{0}\right\|_{\mathrm{E}}^{\alpha} K\left(\left\|\boldsymbol{Z}_{0}\right\|_{\mathrm{E}}^{-1} B^{h} \boldsymbol{Z}\right)\right]=\mathbb{E}\left[\left\|\boldsymbol{Z}_{h}\right\|_{\mathrm{E}}^{\alpha} K\left(\left\|\boldsymbol{Z}_{h}\right\|_{\mathrm{E}}^{-1} \boldsymbol{Z}\right)\right] .
$$

Indeed, (2.6) obviously implies (2.4) and (2.5), (2.5) is obtained by applying (2.4) to the $\alpha$-homogeneous function $H(\boldsymbol{x})=\left\|\boldsymbol{x}_{0}\right\|_{\mathrm{E}}^{\alpha} H_{0}(\boldsymbol{x})$ and (2.6) is obtained by applying (2.4) to the $\alpha$-homogeneous function $H_{0}(x)=\left\|x_{0}\right\|_{\mathrm{E}}^{\alpha} K\left(\left\|x_{0}\right\|_{\mathrm{E}}^{-1} B^{h} \boldsymbol{x}\right)$ defined to be 0 if $\left\|\boldsymbol{x}_{0}\right\|_{\mathrm{E}}=0$.

REMARK 2.5. In the case $E=[0, \infty)^{\mathbb{Z}}$, if $v$ has representation Equation (2.3), then the max-stable process $\boldsymbol{X}$ with exponent measure $\boldsymbol{v}$ defined by (2.1) can be represented as

$$
\left(\boldsymbol{X}_{h}\right)_{h \in \mathbb{Z}} \stackrel{d}{=}\left(\bigvee_{i \geq 1} U_{i} \boldsymbol{Z}_{h}^{(i)}\right)_{h \in \mathbb{Z}},
$$

where $\sum_{i \geq 1} \delta_{U_{i}}$ is a Poisson random measure on $(0, \infty)$ with intensity $\alpha u^{-\alpha-1} \mathrm{~d} u$ and, independently, $\boldsymbol{Z}^{(i)}, i \geq 1$, are independent copies of $\boldsymbol{Z}$. We note in passing that TSF for Brown-Resnick max-stable processes first appears in Dieker and Mikosch [(2015), Lemma 5.2]; see also Hashorva [(2018), Theorem 6.9] for general max-stable processes.

Proof of THEOREM 2.4. It is easily checked that the measure $\boldsymbol{v}$ defined by (2.3) is a tail measure. The condition $\boldsymbol{v}\left(\left\{\mathbf{0}_{\mathrm{E}^{\mathbb{Z}}}\right\}\right)=0$ follows from $\mathbb{P}\left(\boldsymbol{Z}=\mathbf{0}_{\mathrm{E}^{\mathbb{Z}}}\right)=0$. A direct computation yields

$$
\boldsymbol{v}\left(\left\{\left\|\boldsymbol{x}_{h}\right\|_{\mathrm{E}}>1\right\}\right)=\mathbb{E} \int_{0}^{\infty} \mathbb{1}\left\{r\left\|\boldsymbol{Z}_{h}\right\|_{\mathrm{E}}>1\right\} \alpha r^{-\alpha-1} \mathrm{~d} r=\mathbb{E}\left[\left\|\boldsymbol{Z}_{h}\right\|_{\mathrm{E}}^{\alpha}\right],
$$


whence we deduce

$$
\boldsymbol{v}\left\{\left\|\boldsymbol{x}_{0}\right\|_{\mathrm{E}}>1\right\}=\mathbb{E}\left[\left\|\boldsymbol{Z}_{0}\right\|_{\mathrm{E}}^{\alpha}\right]=1, \boldsymbol{v}\left(\left\{\left\|\boldsymbol{x}_{h}\right\|_{\mathrm{E}}>1\right\}\right)=\mathbb{E}\left[\left\|\boldsymbol{Z}_{h}\right\|_{\mathrm{E}}^{\alpha}\right]<\infty .
$$

Homogeneity of order $\alpha$ follows from the simple change of variable $r^{\prime}=u^{-1} r$ : for all $u>0$ and $A \in \mathcal{F}$, we have

$$
\begin{aligned}
\boldsymbol{v}(u A) & =\int_{0}^{\infty} \mathbb{P}(r \boldsymbol{Z} \in u A) \alpha r^{-\alpha-1} \mathrm{~d} r=\int_{0}^{\infty} \mathbb{P}\left(u^{-1} r \boldsymbol{Z} \in u A\right) \alpha r^{-\alpha-1} \mathrm{~d} r \\
& =u^{-\alpha} \int_{0}^{\infty} \mathbb{P}(r \boldsymbol{Z} \in u A) \alpha r^{-\alpha-1} \mathrm{~d} r=u^{-\alpha} \boldsymbol{v}(A) .
\end{aligned}
$$

Conversely, let $\boldsymbol{v}$ be a tail measure and let us prove the existence of a representation (2.3). Let us first prove that there exists at least one measurable functional $\tau$ : $\mathrm{E}^{\mathbb{Z}} \rightarrow[0, \infty)$ having the following properties:

(i) $\tau(\boldsymbol{x})=0$ if and only if $\boldsymbol{x}=\mathbf{0}_{\mathrm{E}^{\mathbb{Z}}}$;

(ii) $\tau$ is 1-homogeneous;

(iii) $\boldsymbol{v}(\{\tau(\boldsymbol{x})>1\})=1$.

Define $p_{h}=\boldsymbol{v}\left(\left\{\left\|\boldsymbol{x}_{h}\right\|_{\mathrm{E}}>1\right\}\right)$ for $h \in \mathbb{Z}$ and let $q \in(0, \infty)^{\mathbb{Z}}$ be a positive sequence such that $\sum_{h \in \mathbb{Z}} p_{h} q_{h}^{\alpha}<\infty$. Consider the map $\tau: \mathbb{E}^{\mathbb{Z}} \rightarrow[0, \infty]$ defined by

$$
\tau(\boldsymbol{x})=\sup _{h \in \mathbb{Z}} q_{h}\left\|\boldsymbol{x}_{h}\right\|_{\mathrm{E}} .
$$

Then $\tau$ is 1-homogeneous and since $\left\|\boldsymbol{x}_{h}\right\|_{\mathrm{E}}=0$ if and only if $\boldsymbol{x}_{h}=\mathbf{0}_{\mathrm{E}}$ for all $h \in \mathbb{Z}$, we have $\tau(\boldsymbol{x})=0$ if and only if $\boldsymbol{x}=\mathbf{0}_{\mathrm{E}} \mathbf{Z}$. By the homogeneity of $\boldsymbol{v}$, we have

$$
\begin{aligned}
& \boldsymbol{v}(\{\tau(\boldsymbol{x})>1\}) \leq \sum_{h \in \mathbb{Z}} \boldsymbol{v}\left(\left\{q_{h}\left\|\boldsymbol{x}_{h}\right\|_{\mathrm{E}}>1\right\}\right)=\sum_{h \in \mathbb{Z}} p_{h} q_{h}^{\alpha}<\infty, \\
& \boldsymbol{v}(\{\tau(\boldsymbol{x})>1\}) \geq q_{0}^{\alpha} \boldsymbol{v}\left(\left\{\left\|\boldsymbol{x}_{0}\right\|_{\mathrm{E}}>1\right\}\right)=q_{0}^{\alpha}>0,
\end{aligned}
$$

whence $\boldsymbol{v}(\{\tau(\boldsymbol{x})>1\}) \in(0, \infty)$. Therefore, by multiplying the sequence $q$ by a suitable normalizing constant, we can impose that $\boldsymbol{v}(\{\tau(\boldsymbol{x})>1\})=1$.

Let now $\tau$ be an arbitrary measurable map having the properties (i), (ii) and (iii) and define the "unit sphere" $S_{\tau}=\{\tau(\boldsymbol{x})=1\}$ and the polar coordinate mapping

$$
\begin{aligned}
T: \mathrm{E}^{\mathbb{Z}} \backslash\left\{\boldsymbol{0}_{\mathrm{E}}\right\} & \rightarrow(0, \infty) \times S_{\tau}, \\
\boldsymbol{x} & \mapsto(\tau(\boldsymbol{x}), \boldsymbol{x} / \tau(\boldsymbol{x})) .
\end{aligned}
$$

Define the probability measure $\sigma$ on $S_{\tau}$ by

$$
\sigma(A)=\boldsymbol{v}(\{\tau(\boldsymbol{x})>1, \boldsymbol{x} / \tau(\boldsymbol{x}) \in A\}), \quad A \in \mathcal{F},
$$

and the measure $v_{\alpha}$ on $(0, \infty)$ with density $\alpha x^{-\alpha-1}$ with respect to Lebesbue measure. Since $T$ is one-to-one and $\tau$ is homogeneous, we obtain the polar representation of $\boldsymbol{v}$, that is $\boldsymbol{v} \circ T^{-1}=v_{\alpha} \otimes \sigma$ or explicitly, for all $A \in \mathcal{F}$,

$$
\boldsymbol{v}(A)=\int_{0}^{\infty} \int_{\mathrm{E}^{\mathbb{Z}}} \mathbb{1}\{r \boldsymbol{x} \in A\} \sigma(\mathrm{d} \boldsymbol{x}) \alpha r^{-\alpha-1} \mathrm{~d} r .
$$


Indeed, starting from the right-hand side of (2.7), we compute

$$
\begin{aligned}
& \int_{0}^{\infty} \int_{E^{\mathbb{Z}}} \mathbb{1}\{r \boldsymbol{x} \in A\} \sigma(\mathrm{d} \boldsymbol{x}) \alpha r^{-\alpha-1} \mathrm{~d} r \\
& \quad=\int_{0}^{\infty} \int_{\mathrm{E}^{\mathbb{Z}}} \mathbb{1}\{\tau(\boldsymbol{x})>1, r \boldsymbol{x} / \tau(\boldsymbol{x}) \in A\} \boldsymbol{v}(\mathrm{d} \boldsymbol{x}) \alpha r^{-\alpha-1} \mathrm{~d} r \\
& \quad=\int_{0}^{\infty} \int_{\mathrm{E}^{\mathbb{Z}}} \tau(\boldsymbol{x})^{-\alpha} \mathbb{1}\{\tau(\boldsymbol{x})>1, r \boldsymbol{x} \in A\} \boldsymbol{v}(\mathrm{d} \boldsymbol{x}) \alpha r^{-\alpha-1} \mathrm{~d} r \\
& \quad=\int_{0}^{\infty} \int_{\mathrm{E}^{\mathbb{Z}}} \tau(\boldsymbol{x})^{-\alpha} \mathbb{1}\{r \tau(\boldsymbol{x})>1, \boldsymbol{x} \in A\} \boldsymbol{v}(\mathrm{d} \boldsymbol{x}) \alpha r^{-\alpha-1} \mathrm{~d} r \\
&=\int_{\mathrm{E}^{\mathbb{Z}}} \mathbb{1}\{\boldsymbol{x} \in A\} \boldsymbol{v}(\mathrm{d} \boldsymbol{x})=\boldsymbol{v}(A) .
\end{aligned}
$$

We use throughout these lines that $\boldsymbol{v}(\{\tau(\boldsymbol{x})=0\})=\boldsymbol{v}\left(\left\{\mathbf{0}_{\mathbb{E} Z}\right\}\right)=0$. The successive equalities rely on the definition of $\sigma$, the changes of variable $r^{\prime}=r / \tau(\boldsymbol{x})$ and $\boldsymbol{x}^{\prime}=$ $\boldsymbol{x} / r$, the homogeneity of $\boldsymbol{v}$ and $\tau$ and finally the fact that $\int_{0}^{\infty} \mathbb{1}\{r>z\} \alpha r^{-\alpha-1} \mathrm{~d} r=$ $z^{-\alpha}$ with $z=1 / \tau(\boldsymbol{x})$.

Consider now a probability space $(\Omega, \mathcal{A}, \mathbb{P})$ on which we can define an $\mathrm{E}^{\mathbb{Z}}$ valued random element $\boldsymbol{Z}$ with distribution $\sigma$. Then (2.7) is exactly the stochastic representation (2.3). The conditions in (2.2) are a consequence of Definition 2.1 together with (2.3): $\boldsymbol{v}\left(\left\{\boldsymbol{0}_{\mathrm{E}} \mathbb{Z}\right\}\right)=\mathbb{P}\left(\boldsymbol{Z}=\mathbf{0}_{\mathrm{E}} \mathbb{Z}\right)=0$ and $\boldsymbol{v}\left(\left\{\left\|\boldsymbol{x}_{h}\right\|_{\mathrm{E}}>1\right\}\right)=\mathbb{E}\left[\left\|\boldsymbol{Z}_{h}\right\|_{\mathrm{E}}^{\alpha}\right]$ is finite for all $h \in \mathbb{Z}$ and equal to 1 for $h=0$.

Finally, assume that $\boldsymbol{v}$ is shift-invariant and let $H_{0}: \mathrm{E}^{\mathbb{Z}} \rightarrow[0, \infty]$ be a 0 homogeneous measurable function. Using the stochastic representation (2.3) and Fubini-Tonelli's theorem for all $h \in \mathbb{Z}$ we obtain

$$
\begin{aligned}
\mathbb{E}\left[\left\|\boldsymbol{Z}_{0}\right\|_{\mathrm{E}}^{\alpha} H_{0}\left(B^{h} \boldsymbol{Z}\right)\right] & =\mathbb{E}\left[H_{0}\left(B^{h} \boldsymbol{Z}\right) \int_{0}^{\infty} \mathbb{1}\left\{r\left\|\boldsymbol{Z}_{0}\right\|_{\mathrm{E}}>1\right\} \alpha r^{-\alpha-1} \mathrm{~d} r\right] \\
& =\int_{\mathbb{E}^{\mathbb{Z}}} H_{0}\left(B^{h} \boldsymbol{x}\right) \mathbb{1}\left\{\left\|\boldsymbol{x}_{0}\right\|_{\mathrm{E}}>1\right\} \boldsymbol{v}(\mathrm{d} \boldsymbol{x}) \\
& =\int_{\mathbb{E}^{\mathbb{Z}}} H_{0}(\boldsymbol{x}) \mathbb{1}\left\{\left\|\boldsymbol{x}_{h}\right\|_{\mathrm{E}}>1\right\} \boldsymbol{v}(\mathrm{d} \boldsymbol{x}) \\
& =\mathbb{E}\left[H_{0}(\boldsymbol{Z}) \int_{0}^{\infty} \mathbb{1}\left\{r\left\|\boldsymbol{Z}_{h}\right\|_{\mathrm{E}}>1\right\} \alpha r^{-\alpha-1} \mathrm{~d} r\right] \\
& =\mathbb{E}\left[H_{0}(\boldsymbol{Z})\left\|\boldsymbol{Z}_{h}\right\|_{\mathrm{E}}^{\alpha}\right] .
\end{aligned}
$$

The third equality uses the shift invariance of $\boldsymbol{v}$ and this proves that (2.5) holds.

Conversely we prove that the tilt shift formula (2.5) implies the shift invariance of $\boldsymbol{v}$. For this purpose, we note that for all $h \in \mathbb{Z}$ and $A \in \mathcal{F}$,

$$
\boldsymbol{v}\left(A \cap\left\{\left\|\boldsymbol{x}_{h}\right\|_{\mathrm{E}}>1\right\}\right)=\int_{0}^{\infty} \mathbb{E}\left[\mathbb{1}\left\{r \boldsymbol{Z} \in A, r\left\|\boldsymbol{Z}_{h}\right\|_{\mathrm{E}}>1\right\}\right] \alpha r^{-\alpha-1} \mathrm{~d} r
$$




$$
\begin{aligned}
& =\int_{1}^{\infty} \mathbb{E}\left[\left\|\boldsymbol{Z}_{h}\right\|_{\mathrm{E}}^{\alpha} \mathbb{1}\left\{r \boldsymbol{Z} /\left\|\boldsymbol{Z}_{h}\right\|_{\mathrm{E}} \in A\right\}\right] \alpha r^{-\alpha-1} \mathrm{~d} r \\
& =\int_{1}^{\infty} \mathbb{E}\left[\left\|\boldsymbol{Z}_{0}\right\|_{\mathrm{E}}^{\alpha} \mathbb{1}\left\{r B^{h} \boldsymbol{Z} /\left\|\boldsymbol{Z}_{0}\right\|_{\mathrm{E}} \in A\right\}\right] \alpha r^{-\alpha-1} \mathrm{~d} r .
\end{aligned}
$$

We used successively the stochastic representation (2.3), the change of variable $r^{\prime}=r\left\|\boldsymbol{Z}_{h}\right\|_{\mathrm{E}}$ [where $\left\|\boldsymbol{Z}_{h}\right\|_{\mathrm{E}}$ is almost surely finite as a consequence of (2.2)] and the tilt-shift formula (2.5). Similarly, for $k \in \mathbb{Z}$,

$$
\begin{aligned}
\left(\boldsymbol{v} \circ B^{-k}\right)\left(A \cap\left\{\left\|\boldsymbol{x}_{h}\right\|_{\mathrm{E}}>1\right\}\right) \\
\quad=\int_{0}^{\infty} \mathbb{E}\left[\mathbb{1}\left\{r B^{k} \boldsymbol{Z} \in A, r\left\|\boldsymbol{Z}_{h-k}\right\|_{\mathrm{E}}>1\right\}\right] \alpha r^{-\alpha-1} \mathrm{~d} r \\
\quad=\int_{1}^{\infty} \mathbb{E}\left[\left\|\boldsymbol{Z}_{h-k}\right\|_{\mathrm{E}}^{\alpha} \mathbb{1}\left\{r B^{k} \boldsymbol{Z} /\left\|\boldsymbol{Z}_{h-k}\right\|_{\mathrm{E}} \in A\right\}\right] \alpha r^{-\alpha-1} \mathrm{~d} r \\
\quad=\int_{1}^{\infty} \mathbb{E}\left[\left\|\boldsymbol{Z}_{0}\right\|_{\mathrm{E}}^{\alpha} \mathbb{1}\left\{r B^{h} \boldsymbol{Z} /\left\|\boldsymbol{Z}_{0}\right\|_{\mathrm{E}} \in A\right\}\right] \alpha r^{-\alpha-1} \mathrm{~d} r .
\end{aligned}
$$

This proves that $\boldsymbol{v}=\boldsymbol{v} \circ B^{-k}$ on the set $\left\{\left\|\boldsymbol{x}_{h}\right\|_{\mathrm{E}}>1\right\}$. Since this holds for all $h \in \mathbb{Z}$, Lemma 2.3 implies $\boldsymbol{v}=\boldsymbol{v} \circ B^{-k}$, whence $\boldsymbol{v}$ is shift-invariant.

2.3. The spectral tail process and the time change formula. The following notion of tail process and spectral tail process plays an important role in the theory of regularly varying time series; see Basrak and Segers (2009). We define here these objects in terms of the tail measure only. The link between these two approaches will be made in Section 3.3 and was already pointed by Owada and Samorodnitsky (2012), Section 4.

DEFINITION 2.6 (Local tail process). Let $\boldsymbol{v}$ be a tail measure on $\mathrm{E}^{\mathbb{Z}}$ and assume that $h \in \mathbb{Z}$ is such that $p_{h}=\boldsymbol{v}\left(\left\{\left\|\boldsymbol{x}_{h}\right\|_{\mathrm{E}}>1\right\}\right)>0$. The local tail process of $\boldsymbol{v}$ at lag $h$ is the process $\boldsymbol{Y}^{(h)}$ with distribution

$$
\mathbb{P}\left(\boldsymbol{Y}^{(h)} \in A\right)=\frac{1}{p_{h}} \boldsymbol{v}\left(\left\{\left\|\boldsymbol{x}_{h}\right\|_{\mathrm{E}}>1, \boldsymbol{x} \in A\right\}\right), \quad A \in \mathcal{F} .
$$

The process $\Theta^{(h)}=\boldsymbol{Y}^{(h)} /\left\|\boldsymbol{Y}_{h}^{(h)}\right\|_{\mathrm{E}}$ is called the local spectral tail process at lag $h$.

For $h=0$, we write simply $\boldsymbol{Y}=\boldsymbol{Y}^{(0)}$ and $\boldsymbol{\Theta}=\boldsymbol{\Theta}^{(0)}$, called the tail process and the spectral tail process associated to $\boldsymbol{v}$.

PROPOSITION 2.7. Let $\boldsymbol{v}$ be a tail measure with stochastic representation (2.3). Then $p_{h}=\boldsymbol{v}\left(\left\{\left\|\boldsymbol{x}_{h}\right\|_{\mathrm{E}}>1\right\}\right)=\mathbb{E}\left[\left\|\boldsymbol{Z}_{h}\right\|_{\mathrm{E}}^{\alpha}\right]<\infty$. If $p_{h}>0$, then $\left\|\boldsymbol{Y}_{h}^{(h)}\right\|_{\mathrm{E}}$ and $\boldsymbol{\Theta}^{(h)}=\boldsymbol{Y}^{(h)} /\left\|\boldsymbol{Y}_{h}^{(h)}\right\|_{\mathrm{E}}$ are independent, $\left\|\boldsymbol{Y}_{h}^{(h)}\right\|_{\mathrm{E}}$ has an $\alpha$-Pareto distribution, that is,

$$
\mathbb{P}\left(\left\|\boldsymbol{Y}_{h}^{(h)}\right\|_{\mathrm{E}}>u\right)=u^{-\alpha}, \quad u>1,
$$


and the distribution of $\boldsymbol{\Theta}^{(h)}$ is given by

$$
\mathbb{P}\left(\boldsymbol{\Theta}^{(h)} \in A\right)=p_{h}^{-1} \mathbb{E}\left[\left\|\boldsymbol{Z}_{h}\right\|_{\mathbb{E}}^{\alpha} \mathbb{1}\left\{\boldsymbol{Z} /\left\|\boldsymbol{Z}_{h}\right\|_{\mathrm{E}} \in A\right\}\right], \quad A \in \mathcal{F} .
$$

PROOF. By definition of the local tail process and using the stochastic representation (2.3), we have for all measurable $H: \mathrm{E}^{\mathbb{Z}} \rightarrow[0, \infty]$,

$$
\begin{aligned}
p_{h} \mathbb{E}\left[H\left(\boldsymbol{Y}^{(h)}\right)\right] & =\int_{\mathbb{E}^{\mathbb{Z}}} H(\boldsymbol{x}) \mathbb{1}\left\{\left\|\boldsymbol{x}_{h}\right\|_{\mathrm{E}}>1\right\} \boldsymbol{v}(\mathrm{d} \boldsymbol{x}) \\
& =\int_{0}^{\infty} \mathbb{E}\left[H(r \boldsymbol{Z}) \mathbb{1}\left\{r\left\|\boldsymbol{Z}_{h}\right\|_{\mathrm{E}}>1\right\}\right] \alpha r^{-\alpha-1} \mathrm{~d} r \\
& =\int_{0}^{\infty} \mathbb{E}\left[H(r \boldsymbol{Z}) \mathbb{1}\left\{r\left\|\boldsymbol{Z}_{h}\right\|_{\mathrm{E}}>1,0<\left\|\boldsymbol{Z}_{h}\right\|<\infty\right\}\right] \alpha r^{-\alpha-1} \mathrm{~d} r \\
& =\int_{1}^{\infty} \mathbb{E}\left[\left\|\boldsymbol{Z}_{h}\right\|_{\mathrm{E}}^{\alpha} H\left(r \boldsymbol{Z} /\left\|\boldsymbol{Z}_{h}\right\|_{\mathrm{E}}\right)\right] \alpha r^{-\alpha-1} \mathrm{~d} r .
\end{aligned}
$$

The last equality relies on the change of variable $r^{\prime}=r\left\|\boldsymbol{Z}_{h}\right\|_{\mathrm{E}}$. Applying this identity with the 0 -homogeneous function $H_{0}(\boldsymbol{x})=\mathbb{1}\left\{\boldsymbol{x} /\left\|\boldsymbol{x}_{h}\right\|_{\mathrm{E}} \in A,\left\|\boldsymbol{x}_{h}\right\|_{\mathrm{E}}>0\right\}$ yields

$$
\begin{aligned}
\mathbb{P}\left(\boldsymbol{\Theta}^{(h)} \in A\right) & =\mathbb{E}\left[H_{0}\left(\boldsymbol{Y}^{(h)}\right)\right]=\int_{1}^{\infty} \mathbb{E}\left[\left\|\boldsymbol{Z}_{h}\right\|_{\mathrm{E}}^{\alpha} H_{0}\left(\boldsymbol{Z} /\left\|\boldsymbol{Z}_{h}\right\|_{\mathrm{E}}\right)\right] \alpha r^{-\alpha-1} \mathrm{~d} r \\
& =p_{h}^{-1} \mathbb{E}\left[\left\|\boldsymbol{Z}_{h}\right\|_{\mathbb{E}}^{\alpha} \mathbb{1}\left\{\boldsymbol{Z} /\left\|\boldsymbol{Z}_{h}\right\|_{\mathrm{E}} \in A\right\}\right],
\end{aligned}
$$

proving Equation (2.9).

COROLLARY 2.8. A tail measure $\boldsymbol{v}$ is shift-invariant if and only if $p_{h}=1$ and $\boldsymbol{\Theta}^{(h)} \stackrel{d}{=} B^{h} \boldsymbol{\Theta}$ for all $h \in \mathbb{Z}$. Then the spectral tail process $\boldsymbol{\Theta}$ characterizes the tail measure $\boldsymbol{v}$ and satisfies

$$
\mathbb{E}\left[H_{0}\left(B^{h} \boldsymbol{\Theta}\right)\right]=\mathbb{E}\left[\left\|\boldsymbol{\Theta}_{h}\right\|_{\mathbb{E}}^{\alpha} H_{0}(\boldsymbol{\Theta})\right], \quad h \in \mathbb{Z},
$$

for all 0-homogeneous measurable $H_{0}: \mathrm{E}^{\mathbb{Z}} \rightarrow[0, \infty)$ vanishing on $\left\{\left\|x_{0}\right\|_{\mathrm{E}}=0\right\}$.

We call Equation (2.10) the time change formula, abbreviated TCF. It first appeared in Basrak and Segers (2009) in the context of stationary regularly varying time series. While the original proof was based on limiting arguments, we propose here a direct proof based on shift invariance of the tail measure, which was already noticed in Owada and Samorodnitsky (2012). In view of Proposition 2.7, the TCF is in fact a direct consequence of the TSF (see the proof below). The condition that $H_{0}$ vanishes on $\left\{\left\|x_{0}\right\|_{\mathrm{E}}=0\right\}$ is important. To stress this, the TCF can be formulated in the equivalent form: for all 0-homogeneous measurable $H_{0}: \mathrm{E}^{\mathbb{Z}} \rightarrow[0, \infty)$,

$$
\mathbb{E}\left[H_{0}\left(B^{h} \boldsymbol{\Theta}\right) \mathbb{1}\left\{\left\|\boldsymbol{\Theta}_{-h}\right\|_{\mathrm{E}}>0\right\}\right]=\mathbb{E}\left[\left\|\boldsymbol{\Theta}_{h}\right\|_{\mathbb{E}}^{\alpha} H_{0}(\boldsymbol{\Theta})\right], \quad h \in \mathbb{Z} .
$$

To see this, simply apply (2.10) to the function $\boldsymbol{x} \mapsto H_{0}(\boldsymbol{x}) \mathbb{1}\left\{\left\|\boldsymbol{x}_{0}\right\|_{\mathrm{E}}>0\right\}$. 
ProOF OF COROLlary 2.8. If $\boldsymbol{v}$ is shift-invariant, then the tilt shift formula (2.5) together with $\mathbb{E}\left[\left\|\boldsymbol{Z}_{0}\right\|_{\mathrm{E}}^{\alpha}\right]=1$ implies $p_{h}=1$ for all $h \in \mathbb{Z}$. Equations (2.5) and (2.9) together imply, for all $h \in \mathbb{Z}, A \in \mathcal{F}$,

$$
\begin{aligned}
\mathbb{P}\left(\boldsymbol{\Theta}^{(h)} \in A\right) & =\mathbb{E}\left[\left\|\boldsymbol{Z}_{h}\right\|_{\mathrm{E}}^{\alpha} \mathbb{1}\left\{\boldsymbol{Z} /\left\|\boldsymbol{Z}_{h}\right\|_{\mathrm{E}} \in A\right\}\right] \\
& =\mathbb{E}\left[\left\|\boldsymbol{Z}_{0}\right\|_{\mathrm{E}}^{\alpha} \mathbb{1}\left\{B^{h} \boldsymbol{Z} /\left\|\boldsymbol{Z}_{0}\right\|_{\mathrm{E}} \in A\right\}\right]=\mathbb{P}\left(B^{h} \boldsymbol{\Theta} \in A\right),
\end{aligned}
$$

whence $\boldsymbol{\Theta}^{(h)} \stackrel{d}{=} B^{h} \boldsymbol{\Theta}$. Conversely, if $p_{h}=1$ and $\boldsymbol{\Theta}^{(h)} \stackrel{d}{=} B^{h} \boldsymbol{\Theta}$ for all $h \in \mathbb{Z}$, then we have for all 0 -homogeneous function $H_{0}$,

$$
\mathbb{E}\left[\left\|\boldsymbol{Z}_{0}\right\|_{\mathbb{E}}^{\alpha} H_{0}\left(B^{h} \boldsymbol{Z}\right)\right]=\mathbb{E}\left[H_{0}\left(B^{h} \boldsymbol{\Theta}\right)\right]=\mathbb{E}\left[H_{0}\left(\boldsymbol{\Theta}^{(h)}\right)\right]=\mathbb{E}\left[\left\|\boldsymbol{Z}_{h}\right\|_{\mathbb{E}}^{\alpha} H_{0}(\boldsymbol{Z})\right],
$$

hence the TSF is satisfied and $\boldsymbol{v}$ is shift-invariant by Theorem 2.4.

If $\boldsymbol{v}$ is shift-invariant, then Equation (2.8) can be rewritten as

$$
\boldsymbol{v}\left(A \cap\left\{\left\|\boldsymbol{x}_{h}\right\|_{\mathrm{E}}>1\right\}\right)=\int_{1}^{\infty} \mathbb{E}\left[\mathbb{1}\left\{r B^{h} \boldsymbol{\Theta} \in A\right\}\right] \alpha r^{-\alpha-1} \mathrm{~d} r,
$$

for all $h \in \mathbb{Z}$ and $A \in \mathcal{F}$. In view of Lemma 2.3, we deduce that $\boldsymbol{\Theta}$ characterizes the shift-invariant tail measure $\boldsymbol{v}$. Furthermore, we have for all 0 -homogeneous function $H_{0}$,

$$
\begin{aligned}
\mathbb{E}\left[\left\|\boldsymbol{\Theta}_{h}\right\|_{\mathrm{E}}^{\alpha} H_{0}(\boldsymbol{\Theta})\right] & =\mathbb{E}\left[\left\|\boldsymbol{Z}_{0}\right\|_{\mathrm{E}}^{\alpha} \frac{\left\|\boldsymbol{Z}_{h}\right\|_{\mathrm{E}}^{\alpha}}{\left\|\boldsymbol{Z}_{0}\right\|_{\mathrm{E}}^{\alpha}} H_{0}\left(\boldsymbol{Z} /\left\|\boldsymbol{Z}_{0}\right\|_{\mathrm{E}}\right)\right] \\
& =\mathbb{E}\left[\left\|\boldsymbol{Z}_{h}\right\|_{\mathrm{E}}^{\alpha} H_{0}(\boldsymbol{Z}) \mathbb{1}\left\{\left\|\boldsymbol{Z}_{0}\right\|_{\mathrm{E}}>0\right\}\right] \\
& =\mathbb{E}\left[\left\|\boldsymbol{Z}_{h}\right\|_{\mathrm{E}}^{\alpha} H_{0}(\boldsymbol{Z})\right]=\mathbb{E}\left[H_{0}\left(B^{h} \boldsymbol{\Theta}\right)\right],
\end{aligned}
$$

where the second line of equalities is valid provided that $H_{0}$ vanishes on $\left\{\left\|x_{0}\right\|_{\mathrm{E}}=\right.$ $0\}$. This shows that if $\boldsymbol{v}$ is shift-invariant, the spectral tail process satisfies the TCF (2.10).

We have introduced the spectral tail process $\boldsymbol{\Theta}$ associated to a tail measure $\boldsymbol{v}$. In the shift-invariant case, it satisfies $\mathbb{P}\left(\left\|\boldsymbol{\Theta}_{0}\right\|_{E}=1\right)=1$ and the TCF (2.10) and it also characterizes $\boldsymbol{v}$. A natural question then arises: if $\boldsymbol{\Theta}$ satisfies $\mathbb{P}\left(\left\|\Theta_{0}\right\|_{E}=1\right)=$ 1 and the TCF, can it be obtained as the spectral tail process of some shift-invariant tail measure $v$ ? In the multivariate setting $\mathrm{E}=\mathbb{R}^{d}$, this question was addressed recently by Janßen (2017) in connection with the theory of max-stable processes. The next theorem still provides a positive answer in the more general framework. Our proofs are different and work directly on the level of the tail measure (not on the level of a stationary regularly varying time series, see Theorem 3.7 below).

THEOREM 2.9. The mapping which to a tail measure associates its spectral tail process is a one-to-one correspondence between the class of shift-invariant tail measures and the class of processes $\boldsymbol{\Theta}$ satisfying $\mathbb{P}\left(\left\|\Theta_{0}\right\|_{\mathrm{E}}=1\right)=1$ and the TCF (2.10). 
PROOF. Starting from a process $\boldsymbol{\Theta}$ satisfying $\mathbb{P}\left(\left\|\boldsymbol{\Theta}_{0}\right\|_{E}=1\right)=1$ and the TCF (2.10), we need to construct a shift-invariant tail measure $\boldsymbol{v}$ with spectral tail process $\boldsymbol{\Theta}$. For $q \in[0, \infty)^{\mathbb{Z}}$ and $\boldsymbol{x} \in \mathrm{E}^{\mathbb{Z}}$, we define

$$
\|\boldsymbol{x}\|_{q, \alpha}=\left(\sum_{j \in \mathbb{Z}} q_{j}\left\|\boldsymbol{x}_{j}\right\|_{\mathrm{E}}^{\alpha}\right)^{1 / \alpha} .
$$

We can always choose the sequence $q$ such that

$$
\mathbb{P}\left(0<\left\|B^{k} \boldsymbol{\Theta}\right\|_{q, \alpha}<\infty\right)=1 \quad \text { for all } k \in \mathbb{Z} .
$$

It suffices to choose $q$ such that $q_{k}>0$ for all $k \in \mathbb{Z}$ and $\sum_{k \in \mathbb{Z}} q_{k}=1$. Then $\mathbb{P}\left(\left\|B^{k} \boldsymbol{\Theta}\right\|_{q, \alpha}>0\right)=1$ since $q_{k}>0$ and $\left\|\boldsymbol{\Theta}_{0}\right\|_{\mathrm{E}}=1$ almost surely. Moreover, applying the time change formula (2.11) with $H \equiv 1$ yields $\mathbb{E}\left[\left\|\Theta_{k}\right\|_{\mathbb{E}}^{\alpha}\right] \leq 1$ for all $k \in \mathbb{Z}$, so that

$$
\mathbb{E}\left[\left\|B^{k} \boldsymbol{\Theta}\right\|_{q, \alpha}^{\alpha}\right]=\sum_{j \in \mathbb{Z}} q_{k} \mathbb{E}\left[\left\|B^{k} \boldsymbol{\Theta}_{j}\right\|_{\mathbb{E}}^{\alpha}\right] \leq \sum_{k \in \mathbb{Z}} q_{k}=1 .
$$

Define $\widetilde{\mathbf{Z}}^{(k)}=B^{k} \boldsymbol{\Theta} /\left\|B^{k} \boldsymbol{\Theta}\right\|_{q, \alpha}, k \in \mathbb{Z}$ and the positive measure $\boldsymbol{v}_{q}$ on $\mathrm{E}^{\mathbb{Z}}$ by

$$
\boldsymbol{v}_{q}(A)=\sum_{k \in \mathbb{Z}} q_{k} \int_{0}^{\infty} \mathbb{P}\left(r \widetilde{\mathbf{Z}}^{(k)} \in A\right) \alpha r^{-\alpha-1} \mathrm{~d} r
$$

for all $A \in \mathcal{F}$. Then $\boldsymbol{v}_{q}$ is obviously $\alpha$-homogeneous and $\boldsymbol{v}_{q}\left(\left\{\mathbf{0}_{\mathrm{E}} \mathbb{Z}\right\}\right)=0$ and we have furthermore, for all measurable function $H: \mathrm{E}^{\mathbb{Z}} \rightarrow[0, \infty)$,

$$
\begin{aligned}
\int_{\mathbb{E}_{\mathbb{Z}}} H(\boldsymbol{x}) \mathbb{1}\left\{\left\|\boldsymbol{x}_{0}\right\|_{\mathrm{E}}>1\right\} \boldsymbol{v}_{q}(\mathrm{~d} \boldsymbol{x}) \\
=\sum_{k \in \mathbb{Z}} q_{k} \int_{0}^{\infty} \mathbb{E}\left[H\left(r \widetilde{\mathbf{Z}}^{(k)}\right) \mathbb{1}\left\{r\left\|\widetilde{\mathbf{Z}}_{0}^{(k)}\right\|_{\mathrm{E}}>1\right\}\right] \alpha r^{-\alpha-1} \mathrm{~d} r \\
=\sum_{k \in \mathbb{Z}} q_{k} \int_{0}^{\infty} \mathbb{E}\left[H\left(\frac{r B^{k} \boldsymbol{\Theta}}{\left\|B^{k} \boldsymbol{\Theta}\right\|_{q, \alpha}}\right) \mathbb{1}\left\{r\left\|\boldsymbol{\Theta}_{-k}\right\|_{\mathrm{E}}>\left\|B^{k} \boldsymbol{\Theta}\right\|_{q, \alpha}\right\}\right] \alpha r^{-\alpha-1} \mathrm{~d} r \\
=\sum_{k \in \mathbb{Z}} q_{k} \int_{1}^{\infty} \mathbb{E}\left[H\left(\frac{r B^{k} \boldsymbol{\Theta}}{\left\|\boldsymbol{\Theta}_{-k}\right\|_{\mathrm{E}}}\right) \frac{\left\|\boldsymbol{\Theta}_{-k}\right\|_{\mathbb{E}}^{\alpha}}{\left\|B^{k} \boldsymbol{\Theta}\right\|_{q, \alpha}^{\alpha}}\right] \alpha r^{-\alpha-1} \mathrm{~d} r \\
=\sum_{k \in \mathbb{Z}} q_{k} \int_{1}^{\infty} \mathbb{E}\left[\left\|\boldsymbol{\Theta}_{-k}\right\|_{\mathbb{E}}^{\alpha} H\left(\frac{r B^{k} \boldsymbol{\Theta}}{\left\|\boldsymbol{\Theta}_{-k}\right\|}\right) \frac{\left\|\boldsymbol{\Theta}_{0}\right\|^{\alpha}}{\| B^{k} \boldsymbol{\Theta}_{q, \alpha}^{\alpha}}\right] \alpha r^{-\alpha-1} \mathrm{~d} r .
\end{aligned}
$$

In these lines, we used successively the definition (2.13), the definition of $\widetilde{\boldsymbol{Z}}$, the change of variable $r^{\prime}=\left\|B^{k} \boldsymbol{\Theta}\right\|_{q, \alpha}\left\|\boldsymbol{\Theta}_{-k}\right\|_{\mathrm{E}}^{-1} r$ (note that the event $\left\{\left\|\boldsymbol{\Theta}_{-k}\right\|_{\mathrm{E}}=0\right\}$ has no contribution to the expectations) and finally the fact that $\mathbb{P}\left(\left\|\Theta_{0}\right\|=1\right)=1$. 
The time change formula now entails

$$
\begin{array}{rl}
\int_{\mathrm{E}^{\mathbb{Z}}} & H(\boldsymbol{x}) \mathbb{1}\left\{\left\|\boldsymbol{x}_{0}\right\|_{\mathrm{E}}>1\right\} \boldsymbol{v}_{q}(\mathrm{~d} \boldsymbol{x}) \\
= & \sum_{k \in \mathbb{Z}} q_{k} \int_{1}^{\infty} \mathbb{E}\left[H\left(\frac{r \boldsymbol{\Theta}}{\left\|\boldsymbol{\Theta}_{0}\right\|_{\mathrm{E}}}\right) \frac{\left\|\boldsymbol{\Theta}_{k}\right\|_{\mathrm{E}}^{\alpha}}{\| \boldsymbol{\Theta}_{q, \alpha}^{\alpha}}\right] \alpha r^{-\alpha-1} \mathrm{~d} r \\
= & \int_{1}^{\infty} \mathbb{E}[H(r \boldsymbol{\Theta})] \alpha r^{-\alpha-1} \mathrm{~d} r .
\end{array}
$$

Applying this identity to the 0-homogeneous function $\boldsymbol{x} \rightarrow H\left(\left\|\boldsymbol{x}_{0}\right\|_{\mathrm{E}}^{-1} \boldsymbol{x}\right) \times$ $\mathbb{1}\left\{\left\|\boldsymbol{x}_{0}\right\|_{\mathrm{E}}>0\right\}$ proves that $\boldsymbol{v}_{q}$ has spectral tail process $\boldsymbol{\Theta}$. It is easily obtained along the same lines, that for all $h \in \mathbb{Z}$,

$$
\int_{\mathrm{E}^{\mathbb{Z}}} H(\boldsymbol{x}) \mathbb{1}\left\{\left\|\boldsymbol{x}_{h}\right\|_{\mathrm{E}}>1\right\} \boldsymbol{v}_{q}(\mathrm{~d} \boldsymbol{x})=\int_{1}^{\infty} \mathbb{E}\left[H\left(r B^{h} \boldsymbol{\Theta}\right)\right] \alpha r^{-\alpha-1} \mathrm{~d} r .
$$

The right-hand side does not depend on $q$ and taking $H \equiv 1$ yields $\boldsymbol{v}_{q}\left(\left\{\left\|\boldsymbol{x}_{h}\right\|_{\mathrm{E}}>\right.\right.$ $1\})=1, h \in \mathbb{Z}$. Therefore, the $\boldsymbol{v}_{q}$ 's are tail measures that coincide on the sets $\left\{\left\|x_{h}\right\|_{\mathrm{E}}>1\right\}, h \in \mathbb{Z}$. By Lemma 2.3, they are all equal, and hence $\boldsymbol{v}_{q}$ does not depend $q$. This entails that $\boldsymbol{v}_{q}$ is shift-invariant since it is readily checked that $\boldsymbol{v}_{q} \circ B^{-h}=\boldsymbol{v}_{B^{h} q}$ whence $\boldsymbol{v}_{q} \circ B^{-h}=\boldsymbol{v}_{q}$.

REMARK 2.10. In two particular cases, a simpler construction of the tail measure corresponding to a given spectral tail process is available.

- If $\mathbb{P}\left(\left\|\boldsymbol{\Theta}_{h}\right\|_{\mathrm{E}}>0\right)=1$ for all $h \in \mathbb{Z}$, then the sequence $q$ can be chosen as $q=\delta_{0}$ and we obtain

$$
\boldsymbol{v}(A)=\int_{0}^{\infty} \mathbb{P}(r \Theta \in A) \alpha r^{-\alpha-1} \mathrm{~d} r .
$$

This provides a stochastic representation (2.3) of $\boldsymbol{v}$ with $\boldsymbol{Z}=\boldsymbol{\Theta}$ such that $\left\|\boldsymbol{Z}_{0}\right\|_{\mathrm{E}}=\left\|\boldsymbol{\Theta}_{0}\right\|_{\mathrm{E}}=1$ almost surely.

- If $\mathbb{P}\left(\sum_{h \in \mathbb{Z}}\left\|\boldsymbol{\Theta}_{h}\right\|_{\mathrm{E}}^{\alpha}<\infty\right)=1$, then we can choose $q \equiv 1$ which yields

$$
\boldsymbol{v}(A)=\sum_{h \in \mathbb{Z}} \int_{0}^{\infty} \mathbb{P}\left(r B^{h} \widetilde{\boldsymbol{Z}} \in A\right) \alpha r^{-\alpha-1} \mathrm{~d} r,
$$

with $\widetilde{\boldsymbol{Z}}=\boldsymbol{\Theta} /\left(\sum_{k \in \mathbb{Z}}\left\|\boldsymbol{\Theta}_{k}\right\|_{\mathrm{E}}^{\alpha}\right)^{1 / \alpha}$. This representation is related to the mixed moving maximum representation of max-stable process; see, for example, Dombry and Kabluchko (2017) and Section 2.5.

We will later need the following lemma on the support of a tail measure. We say that a set $C \in \mathcal{F}$ is a cone if $\boldsymbol{x} \in C$ implies $u \boldsymbol{x} \in C$ for all $u>0$.

LEMMA 2.11. Let $\boldsymbol{v}$ be a tail measure which admits the stochastic representation (2.3). Let $C$ be a cone. Then $\boldsymbol{v}(C)=0 \Leftrightarrow \mathbb{P}(\boldsymbol{Z} \in C)=0$. If $\boldsymbol{v}$ and $C$ are shift-invariant, then $\boldsymbol{v}(C)=0 \Leftrightarrow \mathbb{P}(\Theta \in C)=0$. 
PROOF. If $C$ is a cone, then Equation (2.3) yields $\boldsymbol{v}(C)=\mathbb{P}(\boldsymbol{Z} \in C) \times \infty$, which proves the first statement.

If $\boldsymbol{v}$ is shift-invariant with spectral tail process $\boldsymbol{\Theta}$ and $C$ is a shift invariant cone, the representation (2.13) yields

$$
\begin{aligned}
\boldsymbol{v}(C) & =\sum_{k \in \mathbb{Z}} \int_{0}^{\infty} \mathbb{P}\left(r B^{k} \boldsymbol{\Theta} /\left\|B^{k} \boldsymbol{\Theta}\right\|_{q, \alpha} \in C\right) \alpha r^{-\alpha-1} \mathrm{~d} r \\
& =\sum_{k \in \mathbb{Z}} \int_{0}^{\infty} \mathbb{P}(\boldsymbol{\Theta} \in C) \alpha r^{-\alpha-1} \mathrm{~d} r=\mathbb{P}(\boldsymbol{\Theta} \in C) \times \infty .
\end{aligned}
$$

This proves the second statement.

2.4. Another representation of the tail measure. We propose here another construction proof of Theorem 2.9. It is based on the infargmax functional $I$ defined on $\mathrm{E}^{\mathbb{Z}}$ by

$$
I(\boldsymbol{x})= \begin{cases}-\infty & \text { if } \limsup _{k \rightarrow-\infty}\left\|\boldsymbol{x}_{k}\right\|_{\mathrm{E}}=\sup _{k \in \mathbb{Z}}\left\|\boldsymbol{x}_{k}\right\|_{\mathrm{E}}, \\ j \in \mathbb{Z} & \text { if } \sup _{k \leq j-1}\left\|\boldsymbol{x}_{k}\right\|_{\mathrm{E}}<\left\|\boldsymbol{x}_{j}\right\|_{\mathrm{E}}=\sup _{k \in \mathbb{Z}}\left\|\boldsymbol{x}_{k}\right\|_{\mathrm{E}}, \\ +\infty & \text { if } \sup _{k \leq j}\left\|\boldsymbol{x}_{k}\right\|_{\mathrm{E}}<\sup _{k \in \mathbb{Z}}\left\|\boldsymbol{x}_{k}\right\|_{\mathrm{E}} \text { for all } j .\end{cases}
$$

For $\boldsymbol{x} \in \mathrm{E}^{\mathbb{Z}} \backslash\left\{\boldsymbol{0}_{\mathrm{E}} \mathbb{Z}\right\}$, a sufficient condition for $I(\boldsymbol{x}) \in \mathbb{Z}$ is $\lim _{|k| \rightarrow \infty}\left\|\boldsymbol{x}_{k}\right\|_{\mathrm{E}}=0$.

For two sequences $q \in[0, \infty)^{\mathbb{Z}}$ and $\boldsymbol{x} \in \mathrm{E}^{\mathbb{Z}}$, we define the pointwise multiplication $q \cdot \boldsymbol{x}$ by $(q \cdot \boldsymbol{x})_{k}=q_{k} \boldsymbol{x}_{k}, k \in \mathbb{Z}$.

PROPOSITION 2.12. Let $\boldsymbol{\Theta}$ be a process which satisfies the time change formula (2.10) and let $q \in(0, \infty)^{\mathbb{Z}}$ be such that $\mathbb{P}(I(q \cdot \boldsymbol{\Theta}) \in \mathbb{Z})=1$. Define the measure $\boldsymbol{v}_{q}$ on $\mathrm{E}^{\mathbb{Z}}$ by

$$
\boldsymbol{v}_{q}(A)=\sum_{j \in \mathbb{Z}} \int_{0}^{\infty} \mathbb{P}\left(r B^{j} \boldsymbol{\Theta} \in A, I\left(q \cdot B^{j} \boldsymbol{\Theta}\right)=j\right) \alpha r^{-\alpha-1} \mathrm{~d} r, \quad A \in \mathcal{F} .
$$

Then $\boldsymbol{v}_{q}$ does not depend on $q$ and defines a shift-invariant tail measure with tail spectral process $\boldsymbol{\Theta}$.

Note that any $q \in(0, \infty)^{\mathbb{Z}}$ such that $\sum_{k \in \mathbb{Z}} q_{k}^{\alpha}<\infty$ satisfies $\mathbb{P}(I(q \cdot \boldsymbol{\Theta}) \in$ $\mathbb{Z})=1$. Indeed, the time change formula implies that $\mathbb{E}\left[\left\|\Theta_{h}\right\|_{\mathrm{E}}^{\alpha}\right] \leq 1, h \in \mathbb{Z}$, so that $\mathbb{E}\left[\sum_{j \in \mathbb{Z}} q_{j}^{\alpha}\left\|\boldsymbol{\Theta}_{j}\right\|_{\mathrm{E}}^{\alpha}\right]<\infty$ and therefore $\lim _{|j| \rightarrow \infty} q_{j}\left\|\boldsymbol{\Theta}_{j}\right\|_{\mathrm{E}}=0$ and $I(q \cdot \boldsymbol{\Theta}) \in \mathbb{Z}$ almost surely.

PROOF. It is straightforward to check that $\boldsymbol{v}_{q}$ is $\alpha$-homogeneous and satisfies $\boldsymbol{v}_{q}\left(\left\{\boldsymbol{0}_{\mathrm{E}^{\mathbb{Z}}}\right\}\right)=0$. For all measurable function $H: \mathbb{E}^{\mathbb{Z}} \rightarrow[0, \infty)$, we have

$$
\int_{\mathrm{E}^{\mathbb{Z}}} H(\boldsymbol{x}) \mathbb{1}\left\{\left\|\boldsymbol{x}_{0}\right\|_{\mathrm{E}}>1\right\} \boldsymbol{v}_{q}(\mathrm{~d} \boldsymbol{x})
$$




$$
\begin{aligned}
& =\sum_{j \in \mathbb{Z}} \int_{0}^{\infty} \mathbb{E}\left[H\left(r B^{j} \boldsymbol{\Theta}\right) \mathbb{1}\left\{r\left\|\boldsymbol{\Theta}_{-j}\right\|_{\mathrm{E}}>1, I\left(q \cdot B^{j} \boldsymbol{\Theta}\right)=j\right\}\right] \alpha r^{-\alpha-1} \mathrm{~d} r \\
& =\sum_{j \in \mathbb{Z}} \int_{1}^{\infty} \mathbb{E}\left[\left\|\boldsymbol{\Theta}_{-j}\right\|_{\mathrm{E}}^{\alpha} H\left(\frac{r B^{j} \boldsymbol{\Theta}}{\left\|\boldsymbol{\Theta}_{-j}\right\|_{\mathrm{E}}}\right) \mathbb{1}\left\{I\left(q \cdot B^{j} \boldsymbol{\Theta}\right)=j\right\}\right] \alpha r^{-\alpha-1} \mathrm{~d} r \\
& =\sum_{j \in \mathbb{Z}} \int_{1}^{\infty} \mathbb{E}\left[H\left(\frac{r \boldsymbol{\Theta}}{\left\|\boldsymbol{\Theta}_{0}\right\|_{\mathrm{E}}}\right) \mathbb{1}\{I(q \cdot \boldsymbol{\Theta})=j\}\right] \alpha r^{-\alpha-1} \mathrm{~d} r \\
& =\int_{1}^{\infty} \mathbb{E}\left[H(r \boldsymbol{\Theta}) \sum_{j \in \mathbb{Z}} \mathbb{1}\{I(q \cdot \boldsymbol{\Theta})=j\}\right] \alpha r^{-\alpha-1} \mathrm{~d} r \\
& =\int_{1}^{\infty} \mathbb{E}[H(r \boldsymbol{\Theta})] \alpha r^{-\alpha-1} \mathrm{~d} r .
\end{aligned}
$$

We used here the definition of $\boldsymbol{v}_{q}$ from Proposition 2.12, the change of variable $r^{\prime}=r /\left\|\Theta_{-j}\right\|_{E}$ (note that the event $\left\{\left\|\Theta_{-j}\right\|_{E}=0\right\}$ has no contribution to the integrals), the time change formula, the fact that $\mathbb{P}\left(\left\|\Theta_{0}\right\|_{E}=1\right)=1$ and finally the assumption $\sum_{j \in \mathbb{Z}} \mathbb{P}(I(q \cdot \boldsymbol{\Theta})=j)=1$.

At this point, we have retrieved Equation (2.14) and the remainder of the proof follows exactly the same lines as the proof of Theorem 2.9.

REMARK 2.13. In the particular case $\mathbb{P}(I(\Theta) \in \mathbb{Z})=1$, we can take $q \equiv 1$ and we get

$$
\boldsymbol{v}(A)=\sum_{j \in \mathbb{Z}} \int_{0}^{\infty} \mathbb{P}\left(r B^{j} \boldsymbol{\Theta} \in A, I(\boldsymbol{\Theta})=0\right) \alpha r^{-\alpha-1} \mathrm{~d} r, \quad A \in \mathcal{F} .
$$

Introducing the process $\boldsymbol{Q}$ such that $\mathcal{L}(\boldsymbol{Q})=\mathcal{L}(\boldsymbol{\Theta} \mid I(\boldsymbol{\Theta})=0)$, we obtain

$$
\boldsymbol{v}(A)=\mathbb{P}(I(\boldsymbol{\Theta})=0) \sum_{j \in \mathbb{Z}} \int_{0}^{\infty} \mathbb{P}\left(r B^{j} \boldsymbol{Q} \in A\right) \alpha r^{-\alpha-1} \mathrm{~d} r, \quad A \in \mathcal{F} .
$$

This representation is similar as the one from Equation (2.15). In fact, this is a special case of a moving shift representation of $\boldsymbol{v}$; see Section 2.5.

2.5. Moving shift representations and dissipative tail measures. We consider in this section the relationship between the existence of a moving shift representation and the dissipative/dissipative decomposition of a tail measure. Note that ergodic properties of tail measures are also considered in Owada and Samorodnitsky (2012), Section 5. We introduce only the minimum amount of ergodic theory and define the notion of dissipative tail measure. For more details on (infinite measure) ergodic theory, we refer to Aaronson (1997). The $\sigma$-field on $\mathrm{E}^{\mathbb{Z}}$ generated by cones, or equivalently by 0 -homogeneous functions, is denoted by $\mathcal{C}$. 
DEFINITION 2.14. The dynamical system $\left(\mathrm{E}^{\mathbb{Z}}, \mathcal{C}, \boldsymbol{v}, B\right)$ is said dissipative if there exists a cone $C_{0} \in \mathcal{C}$ such that the sets $B^{h} C_{0}, h \in \mathbb{Z}$, are pairwise disjoint and $\boldsymbol{v}$ is supported by $D=\bigcup_{h \in \mathbb{Z}} B^{h} C_{0}$, that is, $\boldsymbol{v}\left(\mathbb{E}^{\mathbb{Z}} \backslash D\right)=0$.

On the other hand, Remarks 2.10 and 2.13 above motivate the following definition.

DEFINITION 2.15. We say that a shift-invariant tail measure $v$ has a moving shift representation if there exists a stochastic process $\widetilde{Z}$ such that

$$
\boldsymbol{v}(A)=\sum_{h \in \mathbb{Z}} \int_{0}^{\infty} \mathbb{P}\left(r B^{h} \widetilde{\boldsymbol{Z}} \in A\right) \alpha r^{-\alpha-1} \mathrm{~d} r, \quad A \in \mathcal{F} .
$$

The conditions $\boldsymbol{v}\left(\left\{\mathbf{0}_{\mathrm{E}^{\mathbb{Z}}}\right\}\right)=0$ and $\boldsymbol{v}\left(\left\{\left\|\boldsymbol{x}_{0}\right\|_{\mathrm{E}}>1\right\}\right)=1$ entail

$$
\mathbb{P}\left(\widetilde{\boldsymbol{Z}}=\mathbf{0}_{\mathrm{E}^{\mathbb{Z}}}\right)=0, \sum_{h \in \mathbb{Z}} \mathbb{E}\left[\left\|\widetilde{\boldsymbol{Z}}_{h}\right\|_{\mathrm{E}}^{\alpha}\right]=1 .
$$

Indeed, we have

$$
\boldsymbol{v}\left(\left\{\left\|\boldsymbol{x}_{0}\right\|_{\mathrm{E}}>1\right\}\right)=\sum_{h \in \mathbb{Z}} \int_{0}^{\infty} \mathbb{P}\left(r\left\|\widetilde{\boldsymbol{Z}}_{-h}\right\|_{\mathrm{E}}>1\right) \alpha r^{-\alpha-1} \mathrm{~d} r=\sum_{h \in \mathbb{Z}} \mathbb{E}\left[\left\|\widetilde{\boldsymbol{Z}}_{h}\right\|_{\mathrm{E}}^{\alpha}\right] .
$$

Conversely, it is easily proved that, for any stochastic process $\widetilde{Z}$ satisfying (2.17), the measure $\boldsymbol{v}$ defined by (2.16) is a shift-invariant tail measure.

REMARK 2.16. Definition 2.15 is strongly related to the notion of mixed moving maximum representation for max-stable process. If a max-stable process $\boldsymbol{X}$ has a dissipative exponent measure with representation (2.16), then it can be represented as

$$
\boldsymbol{X}_{h} \stackrel{d}{=} \bigvee_{i \geq 1} U_{i} \widetilde{\boldsymbol{Z}}_{h-T_{i}}^{(i)}, \quad h \in \mathbb{Z},
$$

where $\sum_{i \geq 1} \delta_{\left(U_{i}, T_{i}\right)}$ is a Poisson random measure on $(0, \infty) \times \mathbb{Z}$ with intensity equal to the product of $\alpha u^{-\alpha-1} \mathrm{~d} u$ with the counting measure on $\mathbb{Z}$, and, independently, $\widetilde{\boldsymbol{Z}}^{(i)}$ are independent copies of $\widetilde{\boldsymbol{Z}}$. This is a mixed moving maximum representation and $\boldsymbol{X}$ is generated by a dissipative flow [Dombry and Kabluchko (2017), Theorem 8].

REMARK 2.17. Theorem 3.7 states that any tail measure has a stochastic representation (2.3). One can wonder what is a stochastic representation for a tail measure $v$ given by a moving shift representation (2.16). A possible construction is as follows: starting from $\widetilde{\boldsymbol{Z}}$, consider an independent $\mathbb{Z}$-valued random variable $K$ such that $p_{k}=\mathbb{P}(K=k) \in(0,1), k \in \mathbb{Z}$ and define

$$
\boldsymbol{Z}=\sum_{k \in \mathbb{Z}} p_{k}^{-1 / \alpha} B^{k} \widetilde{\boldsymbol{Z}} \mathbb{1}\{K=k\}
$$


In this construction, $\boldsymbol{Z}$ appears as a randomly shifted and rescaled version of $\widetilde{\boldsymbol{Z}}$. It is easy to check that the stochastic representation (2.3) and the dissipative representation (2.16) define the same tail measure $\boldsymbol{v}$.

The converse is not true, that is, a shift-invariant tail measure does not always have a moving shift representation of the form (2.16). The next result is strongly related to Dombry and Kabluchko (2017), Theorem 3. We say that $\boldsymbol{v}$ (resp., $\boldsymbol{Z}, \boldsymbol{\Theta}$ ) is supported by $A \in \mathcal{F}$ if $\boldsymbol{v}\left(A^{c}\right)=0$ with $A^{c}$ the complement of $A$ in $\mathrm{E}^{\mathbb{Z}}$ [resp., $\left.\mathbb{P}\left(\boldsymbol{Z} \in A^{c}\right)=0, \mathbb{P}\left(\boldsymbol{\Theta} \in A^{c}\right)=0\right]$.

Proposition 2.18. Let $\boldsymbol{v}$ be a shift-invariant tail measure. The following statements are equivalent:

(i) $\left(\mathrm{E}^{\mathbb{Z}}, \mathcal{C}, \boldsymbol{v}, B\right)$ is dissipative;

(ii) $v$ has a moving shift representation (2.16);

(iii) $\boldsymbol{v}$ is supported by $\left\{\boldsymbol{x}: \sum_{h \in \mathbb{Z}}\left\|\boldsymbol{x}_{h}\right\|^{\alpha}<\infty\right\}$;

(iv) $\boldsymbol{v}$ is supported by $\left\{\boldsymbol{x}: \lim _{|h| \rightarrow \infty}\left\|\boldsymbol{x}_{h}\right\|_{\mathrm{E}}=0\right\}$;

(v) $\boldsymbol{v}$ is supported by $\{\boldsymbol{x}: I(\boldsymbol{x}) \in \mathbb{Z}\}$.

Proof. 1. (i) $\Rightarrow$ (ii): let $C_{0}$ be as in Definition 2.14. According to Theorem 2.4, there exists an E-valued stochastic process $\boldsymbol{Z}$ which satisfies (2.2) and (2.3). Therefore, the restriction $\boldsymbol{v}_{0}$ of the tail measure $\boldsymbol{v}$ to $C_{0}$ can be represented as

$$
\boldsymbol{v}_{0}(A)=\int_{0}^{\infty} \mathbb{P}(r \boldsymbol{Z} \in A) \alpha r^{-\alpha-1} \mathrm{~d} r,
$$

for all measurable sets $A \subset C_{0}$. The fact that $\boldsymbol{v}$ is dissipative implies that $\boldsymbol{v}=$ $\sum_{h \in \mathbb{Z}} \boldsymbol{v}_{0} \circ B^{-h}$, and hence that $\boldsymbol{v}$ admits the representation (2.16) with $\widetilde{\boldsymbol{Z}}=\boldsymbol{Z}$.

2. (ii) $\Rightarrow$ (iii): If $\boldsymbol{v}$ has a dissipative representation (2.16), then $\widetilde{\boldsymbol{Z}}$ satisfies (2.17) and $\mathbb{E}\left[\sum_{h \in \mathbb{Z}}\left\|\widetilde{\boldsymbol{Z}}_{h}\right\|_{\mathrm{E}}^{\alpha}\right]<\infty$ implies that $\widetilde{\boldsymbol{Z}}$ is supported by $\left\{\boldsymbol{x}: \sum_{h \in \mathbb{Z}}\left\|\boldsymbol{x}_{h}\right\|_{\mathrm{E}}^{\alpha}<\infty\right\}$. Then the representation (2.16) implies that this set also supports $\boldsymbol{v}$.

3. (iii) $\Rightarrow$ (iv) $\Rightarrow$ (v): these implications are trivial since $\sum_{h \in \mathbb{Z}}\left\|x_{h}\right\|_{\mathrm{E}}^{\alpha}<\infty$ implies $\lim _{|h| \rightarrow \infty}\left\|\boldsymbol{x}_{h}\right\|_{\mathrm{E}}=0$, which in turn implies $I(\boldsymbol{x}) \in \mathbb{Z}$ for $\boldsymbol{x} \neq \mathbf{0}_{\mathrm{E}} \mathbb{Z}$ [recall $\left.\boldsymbol{v}\left(\left\{\boldsymbol{0}_{\mathrm{E} Z}\right\}\right)=0\right]$.

4. (v) $\Rightarrow$ (i): take $C_{0}=\{\boldsymbol{x}: I(\boldsymbol{x})=0\}$ to check that $\boldsymbol{v}$ is dissipative.

REMARK 2.19. Since the sets $\left\{\boldsymbol{x}: \sum_{h \in \mathbb{Z}}\left\|\boldsymbol{x}_{h}\right\|_{\mathrm{E}}^{\alpha}<\infty\right\},\left\{\boldsymbol{x}: \lim _{|h| \rightarrow \infty}\left\|\boldsymbol{x}_{h}\right\|_{\mathrm{E}}=\right.$ $0\}$ and $\{\boldsymbol{x}: I(\boldsymbol{x}) \in \mathbb{Z}\}$ are shift-invariant cones, Lemma 2.11 implies that (iii), (iv) and (v) can be equivalently expressed with $\boldsymbol{Z}$ or $\boldsymbol{\Theta}$ where $\boldsymbol{Z}$ is a stochastic representation of $\boldsymbol{v}$ as in (2.3) and $\boldsymbol{\Theta}$ is the corresponding spectral tail process. 
2.6. Maximal indices. We introduce in this section the maximal indices of a shift-invariant tail measure $\boldsymbol{v}$ that are closely connected with the extremal indices of regularly varying stationary time series; see Section 3.4 below.

Given an $\alpha$-homogeneous shift-invariant tail measure $v$ and a 1-homogeneous functional $\tau: E^{\mathbb{Z}} \rightarrow[0, \infty]$ such that $\boldsymbol{v}(\{\tau(\boldsymbol{x})>1\})=1$, we define the quantity $\theta_{\tau} \in[0,1]$, called maximal index, by

$$
\theta_{\tau}=\lim _{n \rightarrow \infty} \frac{1}{n} \boldsymbol{v}\left(\left\{\max _{0 \leq h \leq n-1} \tau\left(B^{h} \boldsymbol{x}\right)>1\right\}\right) .
$$

The existence of the limit is a consequence of Fekete's subadditive lemma. The shift invariance of $\boldsymbol{v}$ implies that the sequence $u_{n}=\boldsymbol{v}\left(\left\{\max _{0 \leq h \leq n-1} \tau\left(B^{h} \boldsymbol{x}\right)>1\right\}\right)$, $n \geq 1$, is subadditive. As a consequence, $u_{n} / n$ converge to $\inf _{n \geq 1} u_{n} / n$ and the limit is in $[0,1]$ since the sequence is nonnegative and $u_{1}=1$.

The next result shows that the maximal indices of a dissipative tail measure are positive and provides expressions of the maximal indices in terms of the stochastic representation and the spectral tail process of the tail measure.

Proposition 2.20. Assume that $\boldsymbol{v}$ is dissipative and that the 1-homogeneous measurable function $\tau: \mathrm{E}^{\mathbb{Z}} \rightarrow[0, \infty]$ satisfies $\boldsymbol{v}(\{\tau(\boldsymbol{x})>1\})=1$. Then $\theta_{\tau}>0$ and

$$
\begin{aligned}
\theta_{\tau} & =\mathbb{E}\left[\sup _{h \in \mathbb{Z}} \tau^{\alpha}\left(B^{h} \widetilde{\boldsymbol{Z}}\right)\right]=\mathbb{E}\left[\frac{\sup _{h \in \mathbb{Z}} \tau^{\alpha}\left(B^{h} \boldsymbol{\Theta}\right)}{\sum_{h \in \mathbb{Z}}\left\|\boldsymbol{\Theta}_{h}\right\|_{\mathbb{E}}^{\alpha}}\right] \\
& =\mathbb{P}(I(\boldsymbol{\Theta})=0) \mathbb{E}\left[\sup _{h \in \mathbb{Z}} \tau^{\alpha}\left(B^{h} \boldsymbol{Q}\right)\right],
\end{aligned}
$$

with $\widetilde{Z}$ as in the dissipative representation (2.16), $\Theta$ the spectral tail process of $\boldsymbol{v}$ and $\boldsymbol{Q}$ is a random sequence in $\mathrm{E}^{\mathbb{Z}}$ with distribution $\mathcal{L}(\boldsymbol{\Theta} \mid I(\boldsymbol{\Theta})=0$ ) as in Remark 2.13.

REMARK 2.21. For a dissipative tail measure $\boldsymbol{v}$ and $\tau(\boldsymbol{x})=\left\|\boldsymbol{x}_{0}\right\|_{\mathrm{E}}$, we also have the following identity proved in Planinić and Soulier [(2018), Lemma 3.2]

$$
\theta_{\tau}=\mathbb{P}\left(\sup _{i \geq 1}\left\|\boldsymbol{Y}_{i}\right\|_{\mathrm{E}} \leq 1\right)=\mathbb{P}\left(\sup _{i \geq 1}\left\|\boldsymbol{Y}_{i}\right\|_{\mathrm{E}}>1\right),
$$

where $\boldsymbol{Y}_{i}=Y \boldsymbol{\Theta}_{i}, i \in \mathbb{Z}$ and $Y$ is a Pareto random variable with tail index $\alpha$, independent of the sequence $\left\{\boldsymbol{\Theta}_{j}\right\}$. This means that the maximal index is in this case the candidate extremal index introduced in Basrak and Segers (2009). The link with the usual extremal index will be made in Section 3.

The proof of Proposition 2.20 makes use of the following identity due to Smith and Weissmannn [(1996), Lemma 3.2]: for a summable sequence $\left(u_{h}\right)_{h \in \mathbb{Z}} \in$ $[0, \infty)^{\mathbb{Z}}$,

$$
\lim _{n \rightarrow \infty} \frac{1}{n} \sum_{h \in \mathbb{Z}} \max _{0 \leq k \leq n-1} u_{h+k}=\sup _{h \in \mathbb{Z}} u_{h} .
$$


Proof of Proposition 2.20. Since $\boldsymbol{v}$ is dissipative, we can introduce a dissipative representation (2.16) and write

$$
\begin{aligned}
\boldsymbol{v}\left(\max _{0 \leq k \leq n-1} \tau\left(B^{k} \boldsymbol{x}\right)>1\right) & =\sum_{h \in \mathbb{Z}} \int_{0}^{\infty} \mathbb{P}\left(r \max _{0 \leq k \leq n-1} \tau\left(B^{k+h} \widetilde{\boldsymbol{Z}}\right)>1\right) \alpha r^{-\alpha-1} \mathrm{~d} r \\
& =\sum_{h \in \mathbb{Z}} \mathbb{E}\left[\max _{0 \leq k \leq n-1} \tau^{\alpha}\left(B^{k+h} \widetilde{\boldsymbol{Z}}\right)\right] .
\end{aligned}
$$

For $n=1$, we have in particular $\sum_{h \in \mathbb{Z}} \mathbb{E}\left[\tau^{\alpha}\left(B^{h} \widetilde{\boldsymbol{Z}}\right)\right]=1$ thanks to the normalizing condition $\boldsymbol{v}(\tau(\boldsymbol{x})>1)=1$. This proves that the sequence $u_{h}=\tau^{\alpha}\left(B^{h} \widetilde{\boldsymbol{Z}}\right), h \in \mathbb{Z}$, is almost surely summable and (2.20) implies

$$
\lim _{n \rightarrow \infty} \frac{1}{n} \sum_{h \in \mathbb{Z}} \max _{0 \leq k \leq n-1} \tau^{\alpha}\left(B^{k+h} \widetilde{\boldsymbol{Z}}\right)=\sup _{h \in \mathbb{Z}} \tau^{\alpha}\left(B^{h} \widetilde{\boldsymbol{Z}}\right) \quad \text { almost surely. }
$$

Furthermore, for all $n \geq 1$, the left-hand side in the previous equation is bounded from above by $\sum_{h \in \mathbb{Z}} \tau^{\bar{\alpha}}\left(B^{h} \widetilde{\boldsymbol{Z}}\right)$ which has finite expectation. Lebesgue's dominated convergence theorem implies

$$
\begin{aligned}
\theta_{\tau} & =\lim _{n \rightarrow \infty} \frac{1}{n} \boldsymbol{v}\left(\max _{0 \leq k \leq n-1} \tau\left(B^{k} \boldsymbol{x}\right)>1\right) \\
& =\lim _{n \rightarrow \infty} \frac{1}{n} \sum_{h \in \mathbb{Z}} \mathbb{E}\left[\max _{0 \leq k \leq n-1} \tau^{\alpha}\left(B^{k+h} \widetilde{\boldsymbol{Z}}\right)\right]=\mathbb{E}\left[\sup _{h \in \mathbb{Z}} \tau^{\alpha}\left(B^{h} \widetilde{\boldsymbol{Z}}\right)\right] .
\end{aligned}
$$

This proves the first formula. The second and third expressions of $\theta_{\tau}$ are special cases obtained for $\widetilde{\boldsymbol{Z}}=\boldsymbol{\Theta} /\left(\sum_{k \in \mathbb{Z}}\left\|\boldsymbol{\Theta}_{k}\right\|_{\mathrm{E}}^{\alpha}\right)^{1 / \alpha}$ and $\widetilde{\boldsymbol{Z}}=\mathbb{P}^{1 / \alpha}(I(\boldsymbol{\Theta})=0) \boldsymbol{Q}$; see Remarks 2.10 and 2.13 .

3. Regularly varying time series on a metric space. In this section, we will build a regularly varying time series with a prescribed tail measure. For this purpose, we first recall the most important definitions and properties of $\mathcal{M}_{0}$ convergence and regular variation on a metric space. For the sake of clarity, the results are stated for a general metric space $F$ in Sections 3.1 and 3.2 and we consider the specific case $\mathrm{F}=\mathrm{E}^{\mathbb{Z}}$ in later sections.

3.1. Regular variation on a metric space. We follow here Hult and Lindskog (2006), Section 3. Let (F, d) be a metric space and let $\mathbf{0}_{\mathrm{F}}$ be an element of $\mathrm{F}$. We assume that there exists a continuous map $(s, \boldsymbol{x}) \rightarrow s \boldsymbol{x}$ from $[0, \infty) \times \mathrm{F}$ to $\mathrm{F}$ such that for all $\boldsymbol{x} \in \mathrm{F}$ and $s \leq t \in(0, \infty), s(t \boldsymbol{x})=(s t) \boldsymbol{x}, 0 \boldsymbol{x}=\mathbf{0}_{\mathrm{F}}$ and

$$
\mathrm{d}\left(\mathbf{0}_{\mathrm{F}}, s \boldsymbol{x}\right) \leq \mathrm{d}\left(\mathbf{0}_{\mathrm{F}}, t \boldsymbol{x}\right) .
$$

Such a map will be called a distance compatible outer multiplication. We denote the ball with center at $\mathbf{0}_{\mathrm{F}}$ and radius $r \geq 0$ by $B_{r}$. We endow $\mathrm{F}$ with its Borel $\sigma$-field. 
Let $\mathcal{M}_{0}(\mathrm{~F})$ be the set of boundedly finite measures on $\mathrm{F} \backslash\left\{\mathbf{0}_{\mathrm{F}}\right\}$, that is, measures $v$ such that $v(A)<\infty$ for all measurable sets $A$ such that $A \cap B_{r}=\varnothing$ for some $r>0$. Such sets will be called separated from $\mathbf{0}_{\mathrm{F}}$. The null measure will be denoted by $\mathbf{0}_{\mathcal{M}}$. We will say that a sequence $\left\{v_{n}, n \geq 1\right\}$ of measures in $\mathcal{M}_{0}(\mathrm{~F})$ converges in $\mathcal{M}_{0}(\mathrm{~F})$ to a measure $v$, which we will denote by $v_{n} \stackrel{\mathcal{M}_{0}(\mathrm{~F})}{\longrightarrow} v$, if

$$
\lim _{n \rightarrow \infty} v_{n}(A)=v(A),
$$

for all measurable set $A$ separated from $\mathbf{0}_{\mathrm{F}}$ and such that $v(\partial A)=0$. This type of convergence is referred to as weak ${ }^{\#}$ convergence in Daley and Vere-Jones (2003) and simply vague convergence in Kallenberg (2017). For more details on the relationship between these different types of convergence, we refer to Lindskog, Resnick and Roy (2014) or Basrak and Planinić (2018).

By Kallenberg [(2017), Lemma 4.1], $\lim _{n \rightarrow \infty} v_{n} \stackrel{\mathcal{M}_{0}(\mathrm{~F})}{\longrightarrow} v$ if and only if $\lim _{n \rightarrow \infty} v_{n}(f)=v(f)$ for all bounded Lipschitz continuous functions with support separated from zero. Hult and Lindskog (2006) proved that convergence in $\mathcal{M}_{0}(\mathrm{~F})$ is equivalent to weak convergence on the complement of balls centered at $\mathbf{0}_{\mathrm{F}}$. More precisely,

$$
\begin{aligned}
v_{n} & \stackrel{\mathcal{M}_{0}(\mathrm{~F})}{\longrightarrow} v \\
& \Longleftrightarrow \text { for all but countably many } r>0, \quad v_{\left.n\right|_{B_{r}^{c}}} \stackrel{w}{\longrightarrow} v_{\left.\right|_{B_{r}^{c}}},
\end{aligned}
$$

where $v_{\left.\right|_{A}}$ is the measure $v$ restricted to the set $A$ and $\stackrel{w}{\rightarrow}$ denotes weak convergence. Convergence in $\mathcal{M}_{0}$ can be metrized. Let $\rho_{r}$ be Prohorov's distance on the set of finite measures defined on $B_{r}^{c}$. Let $\rho$ be the metric on $\mathcal{M}_{0}(\mathrm{~F})$ defined by

$$
\rho(\mu, v)=\int_{0}^{\infty} \mathrm{e}^{-t}\left(\rho_{r}(\mu, v) \wedge 1\right) \mathrm{d} r, \quad \mu, v \in \mathcal{M}_{0}(\mathrm{~F}) .
$$

Then $\left(\mathcal{M}_{0}(\mathrm{~F}), \rho\right)$ is a complete separable metric space; cf. Hult and Lindskog (2006), Theorem 2.3.

We can now define regular varying measures and random elements in $\mathrm{F}$.

\section{DEFINITION 3.1.}

- A Borel measure $\mu$ on $\mathrm{F}$ is said to be regularly varying if there exists a nondecreasing sequence $\left\{a_{n}\right\}$ and a measure $\mu^{*} \in \mathcal{M}_{0}(\mathrm{~F})$ such that $n \mu\left(a_{n} \cdot\right) \stackrel{\mathcal{M}_{0}(\mathrm{~F})}{\longrightarrow} \mu^{*}$. We then write $\mu \in \operatorname{RV}\left(\mathrm{F},\left\{a_{n}\right\}, \mu^{*}\right)$.

- An F-valued random element $X$ defined on a probability space $(\Omega, \mathcal{A}, \mathbb{P})$ is said to be regularly varying if there exists a nondecreasing sequence $\left\{a_{n}\right\}$ tending to infinity and a nonzero measure $v$ on $\mathrm{F} \backslash\left\{\mathbf{0}_{\mathrm{F}}\right\}$ such that $n \mathbb{P}\left(a_{n}^{-1} \boldsymbol{X} \in \cdot\right) \stackrel{\mathcal{M}_{0}(\mathrm{~F})}{\longrightarrow} v$. We then write $\boldsymbol{X} \in \mathrm{RV}\left(\mathrm{F},\left\{a_{n}\right\}, v\right)$. 
By Hult and Lindskog [(2006), Theorem 3.1], if $\boldsymbol{X} \in \mathrm{RV}\left(\mathrm{F},\left\{a_{n}\right\}, v\right)$, then there exists $\alpha>0$ which will be called the tail index of $\boldsymbol{X}$ such that the measure $v$ is $\alpha$ homogeneous and the sequence $\left\{a_{n}\right\}$ is regularly varying with index $1 / \alpha$. We will need the following result which is a straightforward application of the mapping theorem Hult and Lindskog (2006), Theorem 2.5.

LEMMA 3.2. Let $(\mathrm{F}, d)$ and $\left(\mathrm{F}^{\prime}, d^{\prime}\right)$ be two complete separable metric spaces each endowed with a distance compatible outer multipication. Let $\mathbf{0}_{\mathrm{F}} \in \mathrm{F}$ and let $T: \mathrm{F} \rightarrow \mathrm{F}^{\prime}$ be a 1-homogeneous map such that $T\left(\mathbf{0}_{\mathrm{F}}\right)=\mathbf{0}_{\mathrm{F}^{\prime}}$. Set $\mathrm{F}_{0}=\mathrm{F} \backslash\left\{\mathbf{0}_{\mathrm{F}}\right\}$ and $\mathrm{F}_{0}^{\prime}=\mathrm{F}^{\prime} \backslash\left\{\mathbf{0}_{\mathrm{F}^{\prime}}\right\}$. Let $\mu, \mu^{*}$ be a Borel measures on $\mathrm{F}$ and let $\left\{a_{n}\right\}$ be a nondecreasing sequence such that $\mu \in \mathrm{RV}\left(\mathrm{F}_{0}, a_{n}, \mu^{*}\right)$. If $T$ is $\mu^{*}$ almost surely continuous, continuous at $\mathbf{0}_{\mathrm{F}}$, and $\mu^{*} \circ T^{-1}$ is not the null measure, then $\mu \circ T^{-1} \in$ $\mathrm{RV}\left(\mathrm{F}_{0}^{\prime}, a_{n}, \mu^{*} \circ T^{-1}\right)$.

PROOF. Define $\mu_{n}=n \mu\left(a_{n} \cdot\right)$. By assumption, $\mu_{n} \stackrel{\mathcal{M}_{0}(\mathrm{~F})}{\longrightarrow} \mu^{*}$. By homogeneity of $T, \mu_{n} \circ T^{-1}=n \mu \circ T^{-1}\left(a_{n} \cdot\right)$. We want to apply Hult and Lindskog [(2006), Theorem 2.5] to prove that $\mu_{n} \circ T^{-1} \stackrel{\mathcal{M}_{0}\left(\mathrm{~F}^{\prime}\right)}{\longrightarrow} \mu^{*} \circ T^{-1}$. Since $T\left(\mathbf{0}_{\mathrm{F}}\right)=\mathbf{0}_{\mathrm{F}^{\prime}}$, there only remain to prove that if $A$ is bounded away from $\mathbf{0}_{\mathrm{F}^{\prime}}$, then $T^{-1}(A)$ is bounded away from $\mathbf{0}_{\mathrm{F}}$. If $A \subset \mathrm{F}^{\prime}$ is bounded away from $\mathbf{0}_{\mathrm{F}^{\prime}}$, there exists $\varepsilon>0$ such that $\boldsymbol{y} \in$ $A$ implies $\mathrm{d}\left(\boldsymbol{y}, \mathbf{0}_{\mathrm{F}^{\prime}}\right)>\varepsilon$. Since $T$ is continuous and $T\left(\mathbf{0}_{\mathrm{F}}\right)=\mathbf{0}_{\mathrm{F}^{\prime}}$, there exists $\eta>0$ such that $\mathrm{d}\left(\boldsymbol{x}, \mathbf{0}_{\mathrm{F}}\right) \leq \eta$ implies $\mathrm{d}\left(T(\boldsymbol{x}), \mathbf{0}_{\mathrm{F}^{\prime}}\right) \leq \varepsilon$. This proves that if $\boldsymbol{x} \in T^{-1}(A)$ then $\mathrm{d}\left(\boldsymbol{x}, \mathbf{0}_{\mathrm{F}}\right)>\eta$.

3.2. Regular varying Poisson point processes. Let $\mathcal{N}_{0}(\mathrm{~F})$ be the set of boundedly finite point measures on $\mathrm{F} \backslash\left\{\mathbf{0}_{\mathrm{F}}\right\}$, that is, measures $v$ such that $v(A) \in \mathbb{N}$ for all bounded Borel set $A$ separated from $\mathbf{0}_{\mathrm{F}}$. This implies that $v$ has a finite number of points outside each ball centered at $\mathbf{0}_{\mathrm{F}}$ and we can write $v=\sum_{j \geq 1} \delta_{\boldsymbol{x}_{j}}$ where the points of $v$ are numbered in such a way that

$$
d\left(\mathbf{0}_{\mathrm{F}}, \boldsymbol{x}_{i}\right) \geq d\left(\mathbf{0}_{\mathrm{F}}, \boldsymbol{x}_{j}\right)
$$

if $i \leq j$. It is then easily seen that $\mathcal{N}_{0}(\mathrm{~F})$ is a closed subset of $\mathcal{M}_{0}(\mathrm{~F})$ and that the convergence $v_{n} \stackrel{\mathcal{M}_{0}(\mathrm{~F})}{\longrightarrow} v$ implies the convergence of points in $\mathrm{F}$.

The restriction of the distance $\rho$ defined in (3.2) to the space $\mathcal{N}_{0}(\mathrm{~F})$ has the following property. Let the null measure be denoted by $\mathbf{0}_{\mathcal{M}}$ and let $\pi \in \mathcal{N}_{0}(\mathrm{~F})$. Let the largest distance of a point of $\pi$ to $\mathbf{0}_{\mathrm{F}}$ be denoted by $\|\pi\|_{\mathrm{F}}$, that is,

$$
\|\pi\|_{\mathrm{F}}=\sup _{\boldsymbol{x} \in \pi} d\left(\mathbf{0}_{\mathrm{F}}, \boldsymbol{x}\right) .
$$

If $r>\|\pi\|_{\mathrm{F}}$, then $\pi$ has no point outside $B_{r}$, and thus $\rho_{r}\left(\mathbf{0}_{\mathcal{M}}, \pi\right)=0$. Moreover, by definition of the Prohorov distance,

$$
\rho_{r}\left(\mathbf{0}_{\mathcal{M}}, \pi\right)=\inf \left\{\alpha>0: \pi\left(F \cap B_{r}^{c}\right) \leq \alpha, F \text { closed }\right\}=\pi\left(B_{r}^{c}\right) .
$$


That is, the Prohorov distance of a point measure to the zero measure is its number of points. Therefore, if $r>\|\pi\|_{\mathrm{F}}$, then $\rho_{r}\left(\mathbf{0}_{\mathcal{M}}, \pi\right)=0$. This yields

$$
\rho\left(\mathbf{0}_{\mathcal{M}}, \pi\right)=\int_{0}^{\|\pi\|_{\mathrm{F}}} \mathrm{e}^{-r}\left(\rho_{r}\left(\mathbf{0}_{\mathcal{M}}, \pi\right) \wedge 1\right) \mathrm{d} r \leq\|\pi\|_{\mathrm{F}} .
$$

On the other hand, if $r<\|\pi\|_{\mathrm{F}}$ then $\rho_{r}\left(\mathbf{0}_{\mathcal{M}}, \pi\right) \geq 1$ and $1-\mathrm{e}^{-x} \geq(x \wedge 1) / 2$, thus we have

$$
\rho\left(\mathbf{0}_{\mathcal{M}}, \pi\right) \geq \int_{0}^{\|\| \pi \|_{\mathrm{F}}} \mathrm{e}^{-r} \mathrm{~d} r=\left(1-\mathrm{e}^{-\|\pi\|_{\mathrm{F}}}\right) \geq \frac{1}{2}\left(\|\pi\|_{\mathrm{F}} \wedge 1\right) .
$$

These bounds imply that a subset $A \subset \mathcal{N}_{0}(\mathrm{~F})$ is separated from $\mathbf{0}_{\mathcal{M}}$ if there exists $\varepsilon>0$ such that $\|\pi\|_{\mathrm{F}}>\varepsilon$ for all $\pi \in A$.

We define the multiplication $(t, v) \rightarrow t \cdot v$ for $t \in(0, \infty)$ and $v \in \mathcal{M}_{0}(\mathrm{~F})$ by

$$
t \cdot v(f)=\int_{\mathrm{E}} f(t \boldsymbol{x}) v(\mathrm{~d} \boldsymbol{x})
$$

for all nonnegative measurable functions $f$. If $v=\sum_{j \geq 1} \delta_{x_{i}}$ is a point measure, then $t \cdot v=\sum_{j \geq 1} \delta_{t x_{j}}$. Multiplication is continuous with respect to the product topology. For $\pi \in \mathcal{N}_{0}$ and $0<s<t$,

$$
\rho_{r}\left(\mathbf{0}_{\mathcal{M}}, s \pi\right)=\pi\left(s^{-1} B_{r}^{c}\right) \leq \pi\left(s^{-1} B_{r}^{c}\right)=\rho_{r}\left(\mathbf{0}_{\mathcal{M}}, s \pi\right) .
$$

Therefore, we can define a regularly varying point process on $F \backslash\left\{\mathbf{0}_{\mathrm{F}}\right\}$ as a regularly varying element in $\mathcal{N}_{0}(\mathrm{~F})$ in the sense of Definition 3.1.

THEOREM 3.3. Let $\mu_{0}, \mu \in \mathcal{M}_{0}(\mathrm{~F})$ and $\left\{a_{n}\right\}$ be a nondecreasing sequence such that $a_{n} \rightarrow \infty$ and $n \mu_{0}\left(a_{n} \cdot\right) \stackrel{\mathcal{M}_{0}(\mathrm{~F})}{\longrightarrow} \mu$ as $n \rightarrow \infty$. Let $\Pi$ be a Poisson point measure on $\mathrm{F} \backslash\left\{\mathbf{0}_{\mathrm{F}}\right\}$ with mean measure $\mu_{0}$. Then $\Pi \in \operatorname{RV}\left(\mathcal{N}_{0}(\mathrm{~F}),\left\{a_{n}\right\}, \mu^{*}\right)$ where $\mu^{*}$ is a measure on $\mathcal{N}_{0}(\mathrm{~F}) \backslash\left\{\mathbf{0}_{\mathcal{M}}\right\}$ defined by

$$
\mu^{*}(B)=\int_{\mathrm{F}} \mathbb{1}\left\{\delta_{\boldsymbol{x}} \in B\right\} \mu(\mathrm{d} \boldsymbol{x}),
$$

for all Borel set $B$ of $\mathcal{N}_{0}(\mathrm{~F})$ endowed with the distance $\rho$, and $\delta_{\boldsymbol{x}}$ denotes the Dirac mass at $\boldsymbol{x} \in \mathrm{E}$. If $\mu$ is $\alpha$-homogeneous and $\Pi \sim \operatorname{PPP}(\mu)$, then $\Pi \in$ $\mathrm{RV}\left(\mathcal{N}_{0}(\mathrm{~F}), n^{1 / \alpha}, \mu^{*}\right)$.

Note that the limit measure $\mu^{*}$ is the image of $\mu$ under the injection of $\mathrm{F}$ into $\mathcal{N}_{0}(\mathrm{~F})$ defined by $\boldsymbol{x} \mapsto \delta_{\boldsymbol{x}}$. It is concentrated on the subset of point measures that have exactly one point. The underlying heuristic is that given that $\Pi$ is large [in the sense $d(0, \Pi)>u$ with $u \rightarrow \infty$ ], then $\Pi$ can be approximated by a random point measure with only one large point. This is yet another instance of the so-called single large jump principle. 
PROOF. We need to prove the convergence

$$
n \mathbb{P}\left(\Pi / a_{n} \in \cdot\right) \stackrel{\mathcal{M}_{0}\left(\mathcal{N}_{0}(\mathrm{~F})\right)}{\longrightarrow} \mu^{*} .
$$

By Theorem A.1, the convergence (3.5) holds if

$$
\lim _{n \rightarrow \infty} n\left(\mathbb{E}\left[1-\mathrm{e}^{-\int_{\mathrm{F}} f\left(\boldsymbol{x} / a_{n}\right) \Pi(\mathrm{d} \boldsymbol{x})}\right]\right)=\int_{\mathcal{N}_{0}(\mathrm{~F})}\left(1-\mathrm{e}^{-\int_{\mathrm{F}} f(\boldsymbol{x}) \pi(\mathrm{d} \boldsymbol{x})}\right) \mu^{*}(\mathrm{~d} \pi),
$$

for all continuous function $f: \mathrm{F} \rightarrow[0, \infty)$ vanishing on a neighborhood of $\mathbf{0}_{\mathrm{F}}$. By definition of $\mu^{*}$, the right-hand side of (3.6) is equal to

$$
\int_{\mathcal{N}_{0}(\mathrm{~F})}\left(1-\mathrm{e}^{-\int_{\mathrm{F}} f(\boldsymbol{x}) \pi(\mathrm{d} \boldsymbol{x})}\right) \mu^{*}(\mathrm{~d} \pi)=\int_{\mathrm{F}}\left(1-\mathrm{e}^{-f(\boldsymbol{x})}\right) \mu(\mathrm{d} \boldsymbol{x}) .
$$

On the other hand, since $\Pi$ is a Poisson point process, we have

$$
\begin{aligned}
n\left(\mathbb{E}\left[1-\mathrm{e}^{-\int_{\mathrm{F}} f\left(\boldsymbol{x} / a_{n}\right) \Pi(\mathrm{d} \boldsymbol{x})}\right]\right) & =n\left(1-\exp \left[\int_{\mathrm{F}}\left(\mathrm{e}^{-f\left(\boldsymbol{x} / a_{n}\right)}-1\right) \mu_{0}(\mathrm{~d} \boldsymbol{x})\right]\right) \\
& =n\left(1-\exp \left[n^{-1} \int_{\mathrm{F}}-\left(1-\mathrm{e}^{-f(\boldsymbol{x})}\right) \mu_{n}(\mathrm{~d} \boldsymbol{x})\right]\right),
\end{aligned}
$$

with $\mu_{n}=n \mu_{0}\left(a_{n} \cdot\right)$. The function $1-\mathrm{e}^{-f}$ is nonnegative, bounded and with support separated from zero; moreover, $\mu_{n} \rightarrow \mu$ in $\mathcal{M}_{0}$ by assumption, therefore,

$$
\begin{aligned}
\lim _{n \rightarrow \infty} & n\left(\mathbb{E}\left[1-\mathrm{e}^{-\int_{\mathrm{F}} f\left(\boldsymbol{x} / a_{n}\right) \Pi(\mathrm{d} \boldsymbol{x})}\right]\right) \\
& =\lim _{n \rightarrow \infty} n\left(1-\exp \left[n^{-1} \int_{\mathrm{F}}\left(\mathrm{e}^{-f(\boldsymbol{x})}-1\right) \mu_{n}(\mathrm{~d} \boldsymbol{x})\right]\right) \\
& =\int_{\mathrm{F}}\left(1-\mathrm{e}^{-f(\boldsymbol{x})}\right) \mu(\mathrm{d} \boldsymbol{x}) .
\end{aligned}
$$

This proves the convergence (3.6) and the claimed regular variation of $\Pi$.

3.3. Regularly varying time series. We now introduce the notion of a regularly varying time series. We consider a complete separable metric space $\left(E, d_{E}\right)$ with an element $\mathbf{0}_{\mathrm{E}}$ and we assume that the metrid $\mathrm{d}_{\mathrm{E}}$ has the homogeneity property $\mathrm{d}_{\mathrm{E}}\left(\mathbf{0}_{\mathrm{E}}, s \boldsymbol{x}\right)=s \mathrm{~d}_{\mathrm{E}}\left(\mathbf{0}_{\mathrm{E}}, \boldsymbol{x}\right)$ for all $s>0$ and $\boldsymbol{x} \in \mathrm{E}$. We then define the pseudo norm $\|\boldsymbol{x}\|_{\mathrm{E}}=\mathrm{d}_{\mathrm{E}}\left(\mathbf{0}_{\mathrm{E}}, \boldsymbol{x}\right)$.

Definition 3.4. Let $\boldsymbol{X}=\left\{X_{j}, j \in \mathbb{Z}\right\}$ be a time series with values in E. It is said to be regularly varying if $\left(X_{s}, \ldots, X_{t}\right)$ is regularly varying in $\mathrm{E}^{t-s+1}$ for all $s \leq t \in \mathbb{Z}$.

Owada and Samorodnitsky (2012) proved that if $\boldsymbol{X}$ is regularly varying, then there exists a measure $\boldsymbol{v}$ on $\mathbb{E}^{\mathbb{Z}}$, called the tail measure of $\boldsymbol{X}$, whose finite dimensional projections are the exponent measures $\boldsymbol{v}_{s, t}$ and having the properties of a tail 
measure as introduced in Definition 2.1. If $\boldsymbol{X}$ is stationary, then the tail measure is shift invariant.

Consider the metric $\mathrm{d}_{\mathrm{F}}$ on $\mathrm{F}=\mathrm{E}^{\mathbb{Z}}$ defined by

$$
\mathrm{d}_{\mathrm{F}}(\boldsymbol{x}, \boldsymbol{y})=\sum_{j \in \mathbb{Z}} 2^{-|j|}\left(\mathrm{d}_{\mathrm{E}}\left(\boldsymbol{x}_{j}, \boldsymbol{y}_{j}\right) \wedge 1\right) .
$$

It is proved in Segers, Zhao and Meinguet [(2017), Theorem 4.1] that the regular variation of the time series $\boldsymbol{X}$ in the sense of Definition 3.4 is equivalent to the regular variation of $\boldsymbol{X}$ seen as a random element with values in the complete separable metric space $\left(F, d_{F}\right)$ in the sense of Definition 3.1, that is, $X \in R V\left(F,\left\{a_{n}\right\}, v\right)$ with $a_{n}$ such that $\lim _{n \rightarrow \infty} n \mathbb{P}\left(\left\|\boldsymbol{X}_{0}\right\|_{\mathrm{E}}>a_{n}\right)=1$. Therefore, we will hereafter indifferently say that $\boldsymbol{X}$ is regularly varying in the sense of Definition 3.4 with tail measure $\boldsymbol{v}$ or $\boldsymbol{X} \in \mathrm{RV}\left(\mathrm{E}^{\mathbb{Z}},\left\{a_{n}\right\}, \boldsymbol{v}\right)$.

The local tail process and spectral tail process associated to the tail measure $v$ can be reinterpreted as limiting quantities for the regularly varying time series $\boldsymbol{X}$. Their existence also characterizes regular variation. The next result generalizes Basrak and Segers [(2009), Theoreom 2.1] for a nonstationary time series.

LEMMA 3.5. Let $\boldsymbol{v}$ be a tail measure on $\mathrm{E}^{\mathbb{Z}}$ and for $h \in \mathbb{Z}$ set $p_{h}=\boldsymbol{v}\left(\left\{\|\boldsymbol{x}\|_{\mathrm{E}}>\right.\right.$ $1\})$. For $h$, such that $p_{h}>0$, let $\boldsymbol{Y}^{(h)}$ and $\boldsymbol{\Theta}^{(h)}$ be the local tail and spectral tail processes associated to $\boldsymbol{v}$ as in Definition 2.6. The following statements are equivalent:

(i) $\boldsymbol{X} \in \mathrm{RV}\left(\mathrm{E}^{\mathbb{Z}},\left\{a_{n}\right\}\right.$, v);

(ii) For all $h \in \mathbb{Z}, \lim _{n \rightarrow \infty} n \mathbb{P}\left(\left\|X_{h}\right\|_{\mathrm{E}}>a_{n}\right)=p_{h}$ and for all $h$ such that $p_{h}>0$, we have, as $u \rightarrow \infty$,

$$
\mathcal{L}\left(\boldsymbol{X} / u \mid\left\|\boldsymbol{X}_{h}\right\|_{\mathrm{E}}>u\right) \stackrel{d}{\longrightarrow} \boldsymbol{Y}^{(h)} ;
$$

(iii) For all $h \in \mathbb{Z}, \lim _{n \rightarrow \infty} n \mathbb{P}\left(\left\|X_{h}\right\|_{\mathrm{E}}>a_{n}\right)=p_{h}$ and for $h$ such that $p_{h}>0$,

$$
\mathcal{L}\left(\boldsymbol{X} /\left\|\boldsymbol{X}_{h}\right\|_{\mathrm{E}} \mid\left\|\boldsymbol{X}_{h}\right\|_{\mathrm{E}}>u\right) \stackrel{d}{\longrightarrow} \boldsymbol{\Theta}^{(h)} .
$$

If $\boldsymbol{X}$ is stationary, then $\boldsymbol{\Theta}^{(h)} \stackrel{d}{=} B^{h} \boldsymbol{\Theta}$ and $\boldsymbol{v}$ is shift-invariant.

PROOF. We start by proving the implication (i) $\Rightarrow$ (ii). By definition of regular variation, for every $h \in \mathbb{Z}$ we have $\lim _{n \rightarrow \infty} n \mathbb{P}\left(\left\|X_{h}\right\|_{\mathrm{E}}>a_{n}\right)=p_{h}$ and for every set $A$ depending only on a finite number of coordinates, we have

$$
\lim _{n \rightarrow \infty} n \mathbb{P}\left(\boldsymbol{X} \in A,\left\|\boldsymbol{X}_{h}\right\|_{\mathrm{E}}>a_{n}\right)=\boldsymbol{v}\left(\left\{A \cap\left\{\left\|\boldsymbol{x}_{h}\right\|_{\mathrm{E}}>1\right\}\right) .\right.
$$

By definition of the local tail process, we obtain

$$
\lim _{n \rightarrow \infty} \mathbb{P}\left(\boldsymbol{X} \in A \mid\left\|\boldsymbol{X}_{h}\right\|_{\mathrm{E}}>a_{n}\right)=\frac{1}{p_{h}} \boldsymbol{v}\left(\left\{A \cap\left\{\left\|\boldsymbol{x}_{h}\right\|_{\mathrm{E}}>1\right\}\right)=\mathbb{P}\left(\boldsymbol{Y}^{(h)} \in A\right) .\right.
$$


To prove the converse implication (ii) $\Rightarrow$ (i), we first note that the tail measure is characterized by its finite dimensional projections. Therefore, it suffices to prove that these projections are characterized by the tail process. Let $A$ be a set which depends only on the coordinates between $s$ and $t, s \leq t \in \mathbb{Z}$, and bounded away from zero in $\mathrm{E}^{t-s+1}$. This means that there exists $\varepsilon>0$ such that $x \in A$ implies that $\sum_{h=s}^{t} \mathbb{1}\left\{\|\boldsymbol{x}\|_{\mathrm{E}}>\varepsilon\right\} \geq 1$. Note also that if $p_{h}=0$, then for all $\varepsilon>0$, $\lim _{n \rightarrow \infty} n \mathbb{P}\left(\left\|\boldsymbol{X}_{h}\right\|_{\mathrm{E}}>a_{n} \varepsilon\right)=0$. Thus in the following computations we will omit the indices $h$ such that $p_{h}=0$. Decomposing according to the first exceedance over $\varepsilon a_{n}$, we obtain

$$
\begin{aligned}
\boldsymbol{v}_{s, t}(A)= & \lim _{n \rightarrow \infty} n \mathbb{P}\left(a_{n}^{-1} \boldsymbol{X}_{x, t} \in A\right) \\
= & \lim _{n \rightarrow \infty} \sum_{h=s}^{t} n \mathbb{P}\left(a_{n}^{-1} \boldsymbol{X}_{x, t} \in A,\left\|\boldsymbol{X}_{h}\right\|_{\mathrm{E}}>\varepsilon, \max _{s \leq i<h}\left\|\boldsymbol{X}_{i}\right\|_{\mathrm{E}} \leq \varepsilon\right) \\
= & \lim _{n \rightarrow \infty} \sum_{\substack{h=s \\
p_{h}>0}}^{t} n \mathbb{P}\left(a_{n}^{-1}\left\|\boldsymbol{X}_{h}\right\|_{\mathrm{E}}>a_{n} \varepsilon\right) \\
& \times \frac{\mathbb{P}\left(a_{n}^{-1} \boldsymbol{X}_{x, t} \in A,\left\|\boldsymbol{X}_{h}\right\|_{\mathrm{E}}>\varepsilon, \max _{s \leq i<h}\left\|\boldsymbol{X}_{i}\right\|_{\mathrm{E}} \leq \varepsilon\right)}{\mathbb{P}\left(a_{n}^{-1}\left\|\boldsymbol{X}_{h}\right\|_{\mathrm{E}}>a_{n} \varepsilon\right)} \\
= & \sum_{\substack{h=s \\
p_{h}>0}} \varepsilon^{-\alpha} p_{h} \mathbb{P}\left(\varepsilon \boldsymbol{Y}_{s, t}^{(h)} \in A, \max _{s \leq i \leq h-1}\left\|\boldsymbol{Y}_{i}\right\|_{\mathrm{E}} \leq 1\right) .
\end{aligned}
$$

This proves that the finite dimensional distributions of the tail process characterize the tail measure. The proof of the equivalence (ii) $\Leftrightarrow$ (iii) is straightforward generalization of the corresponding result for $\mathbb{R}^{d}$ valued time series in Basrak and Segers (2009) and is omitted.

REMARK 3.6. In the case $\mathrm{E}=[0, \infty)$, Lemma 3.5 implies that the tail measure of a time series $\boldsymbol{X} \in \operatorname{RV}\left([0, \infty)^{\mathbb{Z}},\left\{a_{n}\right\}, \boldsymbol{v}\right)$ is the exponent measure of the limiting max-stable process, see Remarks 2.2 and 2.5. More precisely, let $\boldsymbol{X}^{(i)}$, $i \geq 1$, be i.i.d. copies of $\boldsymbol{X}$. Then the regular variation of $\boldsymbol{X}$ implies that

$$
a_{n}^{-1} \bigvee_{i=1}^{n} \boldsymbol{X}^{(i)} \stackrel{\text { fi.di. }}{\longrightarrow} \bigvee_{i=1}^{\infty} \mathbf{P}^{(i)},
$$

where the suprema are taken componentwise and $\sum_{i=1}^{\infty} \delta_{\mathbf{P}^{(i)}}$ is a Poisson point process on $[0, \infty)^{\mathbb{Z}}$ with mean measure $\boldsymbol{v}$. This also shows that for a max-stable process the tail measure and the exponent measure are the same.

In the sequel, given a shift-invariant tail measure, or equivalently given a spectral tail process, we will build a time series 
3.3.1. Construction of a stationary regularly varying time series. As seen in Section 2, the tail measure of a stationary regularly varying time series with tail in$\operatorname{dex} \alpha>0$ is a shift-invariant tail measure with homogeneous with index $\alpha$. A natural question is whether any shift-invariant tail measure $v$ on $F=E^{\mathbb{Z}}$ is the tail measure of a stationary regularly varying time series $\boldsymbol{X}$. The purpose of this section is to prove that the answer is positive and provide one construction for such a process $\boldsymbol{X}$.

Our intuition is guided by the case $E=[0, \infty)$. Then, given a tail measure $\boldsymbol{v}$ on $[0, \infty)^{\mathbb{Z}}$, the max-stable process $\boldsymbol{X}$ with exponent measure $\boldsymbol{v}$ is regularly varying with tail measure $\boldsymbol{v}$. Furthermore, $\boldsymbol{X}$ is stationary if and only if $\boldsymbol{v}$ is shift-invariant. This provides a straightforward solution in the nonnegative case. Before we generalize it, we recall the Poisson point process representation of the max-stable process $\boldsymbol{X}$ : if $\boldsymbol{v}$ admits representation (2.3) with $\boldsymbol{Z}$ a nonnegative time series, then

$$
\boldsymbol{X} \stackrel{d}{=} \bigvee_{i \geq 1} \Gamma_{i}^{-1 / \alpha} \boldsymbol{Z}^{(i)}
$$

where $\left\{\Gamma_{i}\right\}_{i \geq 1}$ are the points of a homogeneous Poisson process on $[0, \infty)$ and independently, $\boldsymbol{Z}^{(i)}, i \geq 1$, are independent copies of $\boldsymbol{Z}$ and the supremum is taken componentwise.

In the general framework where $E$ is a complete separable metric space and $v$ is a tail measure on $F=E^{\mathbb{Z}}$, we consider a Poisson point process $\Pi \sim \operatorname{PPP}(v)$. Note $\Pi$ can be constructed as

$$
\Pi=\left\{\Gamma_{i}^{-1 / \alpha} \boldsymbol{Z}^{(i)}, i \geq 1\right\} .
$$

We interpret the point process $\Pi$ as a particle system that evolves in time, the $i$ th particle having position $\varphi_{h}^{(i)}=\Gamma_{i}^{-1 / \alpha} \boldsymbol{Z}_{h}^{(i)}$ at time $h$. The random process $\varphi^{(i)}=$ $\Gamma_{i}^{-1 / \alpha} \boldsymbol{Z}^{(i)} \in \mathrm{F}=\mathrm{E}^{\mathbb{Z}}$ is hence the trajectory of the $i$ th particle. We construct a time series $\boldsymbol{X}$ that records at each time $h$ the position of the particle which is farthest away from $\mathbf{0}_{\mathrm{E}}$, which we will call the largest point. More formally, we define

$$
\boldsymbol{X}_{h}=\boldsymbol{\varphi}_{h}^{\left(i_{h}\right)}, \quad i_{h}=\underset{i \geq 1}{\arg \max }\left\|\varphi_{h}^{(i)}\right\|_{\mathrm{E}}, \quad h \in \mathbb{Z} .
$$

Provided $\mathbb{P}\left(\left\|\boldsymbol{Z}_{h}\right\|_{E}>0\right)>0$, there are almost surely infinitely many particles at time $h$ with positive norm and a unique particle with the largest norm. This is because the random variables $\Gamma_{i}, i \geq 1$ have continuous distributions and $\lim _{i \rightarrow \infty} \Gamma_{i}^{-1 / \alpha}=0$ almost surely. Therefore, the $\arg \max$ in (3.11) is unique and the random variable $i_{h}$ is well defined.

THEOREM 3.7. Given a shift-invariant tail measure $\boldsymbol{v}$ on $\mathrm{E}^{\mathbb{Z}}$, the $\mathrm{E}$-valued time series $\boldsymbol{X}$ defined by (3.11) is stationary and regularly varying on $\mathrm{E}^{\mathbb{Z}}$ with sequence $a_{n}=n^{1 / \alpha}$ and tail measure $\boldsymbol{v}$. 
ProOF. We will use the mapping Lemma 3.2. We consider $F=E^{\mathbb{Z}}$ endowed with the metric $\mathrm{d}_{\mathrm{F}}$ defined in (3.7).

Define the subset $\mathcal{N}_{0}^{\sharp}(E) \subset \mathcal{N}_{0}(E)$ as the set of point measures that have exactly one largest point and consider the map $T: \mathcal{N}_{0}^{\sharp}(\mathrm{E}) \rightarrow \mathrm{E}$ that associate to such a point measure its largest point. By Lemma B.2, $\mathcal{N}_{0}^{\sharp}(E)$ is open and $T$ is continuous on $\mathcal{N}_{0}^{\sharp}(\mathrm{E})$. We extend $T$ to $\mathcal{N}_{0}(\mathrm{E})$ by setting the value $\mathbf{0}_{\mathrm{E}}$ on $\mathcal{N}_{0}(\mathrm{E}) \backslash \mathcal{N}_{0}^{\sharp}(\mathrm{E})$.

Given a point measure $\pi \in \mathcal{N}_{0}(\mathrm{~F})$ and $h \in \mathbb{Z}$, we define $P_{h}(\pi)$ as the restriction to $\mathrm{E} \backslash\left\{\mathbf{0}_{\mathrm{E}}\right\}$ of the image of $\pi$ under the projection $\boldsymbol{x} \mapsto \boldsymbol{x}_{h}$. More precisely, if $\pi=\sum_{i=1}^{\infty} \delta_{\boldsymbol{x}^{(i)}}$ with $\boldsymbol{x}^{(i)} \in \mathrm{F}$, then $P_{h}(\pi)$ is the point measure on $\mathrm{E} \backslash\left\{\boldsymbol{0}_{\mathrm{E}}\right\}$ with points $\boldsymbol{x}_{h}^{(i)}$ such that $\boldsymbol{x}_{h}^{(i)} \neq \mathbf{0}_{\mathrm{E}}$. For the particle system $\Pi=\left\{\varphi^{(i)}, i \geq 1\right\}$,

$$
P_{h} \Pi=\left\{\varphi_{h}^{(i)}: i \geq 1, \varphi_{h}^{(i)} \neq \mathbf{0}_{\mathrm{E}}\right\}
$$

records the position at time $h \in \mathbb{Z}$ of the nonzero particles. Using the representation (3.10), we also have

$$
P_{h} \Pi=\left\{\Gamma_{i}^{-1 / \alpha} \boldsymbol{Z}_{h}^{(i)}: i \geq 1, \boldsymbol{Z}_{h}^{(i)} \neq \mathbf{0}_{\mathrm{E}}\right\}
$$

Since $\Pi$ is Poisson, $P_{h} \Pi$ is a Poisson point process on $\mathrm{E} \backslash\left\{\mathbf{0}_{\mathrm{E}}\right\}$ with intensity

$$
\mu(B)=\boldsymbol{v}\left(\left\{\boldsymbol{x}: \boldsymbol{x}_{h} \in B\right\}\right)=\boldsymbol{v}\left(\left\{\boldsymbol{x}: \boldsymbol{x}_{0} \in B\right\}\right),
$$

for all Borel measurable sets $B \subset \mathrm{E} \backslash\left\{\mathbf{0}_{\mathrm{E}}\right\}$. The marginal measure $\mu$ does not depend on $h \in \mathbb{Z}$ because $\boldsymbol{v}$ is shift-invariant. Moreover, $P_{h} \Pi \in \mathcal{N}_{0}^{\sharp}(E)$ almost surely since for $i \neq j, \mathbb{P}\left(\Gamma_{i}^{-1 / \alpha}\left\|\boldsymbol{Z}_{h}^{(i)}\right\|_{\mathrm{E}}=\Gamma_{j}^{-1 / \alpha}\left\|\boldsymbol{Z}_{h}^{(j)}\right\|_{\mathrm{E}}\right)=0$.

We now define the map $\mathcal{T}$ on $\mathcal{M}_{0}(\mathrm{~F})$ onto $\mathrm{F}$ by

$$
\mathcal{T}(\pi)=\left\{T\left(P_{h} \pi\right), h \in \mathbb{Z}\right\} .
$$

The time series $\boldsymbol{X}$ defined in (3.11) can be reexpressed in terms of the map $\mathcal{T}$ : $\boldsymbol{X}=\mathcal{T}(\Pi)$. The stationarity of $\boldsymbol{X}_{h}$ follows from the shift-invariance of $\boldsymbol{v}$ since $B \boldsymbol{X}=\mathcal{T}(B \Pi) \stackrel{d}{=} \mathcal{T}(\Pi)$ where $B \Pi=\left\{B \varphi^{(i)}, i \geq 1\right\} \stackrel{d}{=} \Pi$. The regular variation of $\boldsymbol{X}$ will be obtained as a consequence of Lemma 3.2. By construction, $\mathcal{T}$ is 1 homogeneous, $\mathcal{T}\left(\mathbf{0}_{\mathcal{M}}\right)=\mathbf{0}_{\mathrm{F}}$ and we will check the following properties:

(a) the map $\mathcal{T}$ is continuous at $\mathbf{0}_{\mathcal{M}}$;

(b) the map $\mathcal{T}$ is almost surely continuous with respect to the distribution of $\Pi$.

- To prove that $\mathcal{T}$ is continuous at $\mathbf{0}_{\mathcal{M}}$, recall that the space $\mathrm{F}$ is endowed with the distance defined in (3.7) and note that for $\pi=\sum_{i=1}^{\infty} \delta_{\boldsymbol{x}^{(i)}} \in \mathcal{N}_{0}(\mathrm{~F})$,

$$
\mathrm{d}_{\mathrm{F}}\left(\mathbf{0}_{\mathrm{F}}, \mathcal{T}(\pi)\right)=\sum_{h \in \mathbb{Z}} 2^{-|h|} \max _{i \geq 1}\left(\left\|\boldsymbol{x}_{h}^{(i)}\right\|_{\mathrm{E}} \wedge 1\right) \leq 3 \max _{i \geq 1} \mathrm{~d}_{\mathrm{F}}\left(\mathbf{0}_{\mathrm{F}}, \boldsymbol{x}^{(i)}\right)=3\|\pi\|_{\mathrm{F}} .
$$

On the other hand, applying (3.4), we obtain that if $\rho\left(\mathbf{0}_{\mathcal{M}}, \pi\right)<1 / 4$, then

$$
\mathrm{d}_{\mathrm{F}}\left(\mathbf{0}_{\mathrm{F}}, \mathcal{T}(\pi)\right) \leq 12 \rho\left(\mathbf{0}_{\mathcal{M}}, \pi\right) .
$$

This proves (a). 
- We now prove (b). By Lemma B.3, it suffices to prove that the projections $T_{h}=$ $T \circ P_{h}$ are continuous for all $h$. Since $\mathbb{P}\left(P_{h} \Pi \in \mathcal{N}_{0}^{\sharp}(\mathrm{E})\right)=1$, this follows from the continuity of $T$ on $\mathcal{N}_{0}^{\sharp}(E)$ which is established in Lemma B.2.

To conclude the proof, there only remains to prove that the tail measure of $\boldsymbol{X}$ is $\boldsymbol{v}$. By Lemma 3.2 and Theorem 3.3, the tail measure of $\boldsymbol{X}$ is $\mu^{*} \circ \mathcal{T}^{-1}$, given for $A \in \mathrm{F} \backslash\left\{\mathbf{0}_{\mathrm{F}}\right\}$ by

$$
\mu^{*} \circ \mathcal{T}^{-1}(A)=\int_{\mathrm{F}} \mathbb{1}\left\{\mathcal{T}\left(\delta_{\boldsymbol{x}}\right) \in A\right\} \boldsymbol{v}(\mathrm{d} \boldsymbol{x}) .
$$

For $\boldsymbol{x}=\left\{\boldsymbol{x}_{h}, h \in \mathbb{Z}\right\} \in \mathrm{F}$, we have $\mathcal{T}\left(\delta_{\boldsymbol{x}}\right)=\left\{T\left(\delta_{\boldsymbol{x}_{h}}\right), h \in \mathbb{Z}\right\}=\boldsymbol{x}$ if $\boldsymbol{x} \neq \mathbf{0}_{\mathrm{F}}$ and $\mathcal{T}\left(\mathbf{0}_{\mathrm{F}}\right)=\mathcal{T}\left(\mathbf{0}_{\mathcal{M}}\right)=\mathbf{0}_{\mathrm{F}}$. Thus $\mu^{*} \circ \mathcal{T}^{-1}=\boldsymbol{v}$.

The next two propositions state some interesting elementary properties of the process $\boldsymbol{X}$ defined by (3.11). They are strongly related to max-stability. Let $g$ : $\mathrm{F} \rightarrow \mathrm{F}$ be the map defined by $g(\boldsymbol{x})=\left\{\left\|\boldsymbol{x}_{h}\right\|_{\mathrm{E}}, h \in \mathbb{Z}\right\}$.

Proposition 3.8. Consider the process $\boldsymbol{X}$ defined by (3.11). Then the nonnegative time series $\left\{\left\|\boldsymbol{X}_{h}\right\|_{\mathrm{E}}, h \in \mathbb{Z}\right\}$ is max-stable with exponent measure $\boldsymbol{v} \circ g^{-1}$.

PROOF. The max stability follows from the representation $\left\|\boldsymbol{X}_{h}\right\|_{\mathrm{E}}=$ $\sup _{i \geq 1} \Gamma_{i}^{-1 / \alpha}\left\|Z_{i}\right\|_{\mathrm{E}}$ and the fact that $\boldsymbol{v} \circ g^{-1}$ is the exponent measure is a consequence of the mapping theorem Lemma 3.2, since for a max-stable process, the tail measure and exponent measure are the same.

In order to study further the stability property of the process $\boldsymbol{X}$, we define the binary operation $\odot$ defined on $E$ by

$$
x_{1} \odot x_{2}=\left\{\begin{array}{ll}
x_{1} & \text { if }\left\|x_{1}\right\|_{\mathrm{E}} \geq\left\|x_{2}\right\|_{\mathrm{E}}, \\
x_{2} & \text { otherwise, }
\end{array} \quad \boldsymbol{x}_{1}, \boldsymbol{x}_{2} \in \mathrm{E} .\right.
$$

Note that the binary operation $\odot$ is associative, that is, $\left(x_{1} \odot x_{2}\right) \odot x_{3}=x_{1} \odot$ $\left(\boldsymbol{x}_{2} \odot \boldsymbol{x}_{3}\right)$ for all $\boldsymbol{x}_{1}, \boldsymbol{x}_{2}, \boldsymbol{x}_{3} \in \mathrm{E}$. It is not commutative since $\boldsymbol{x}_{1} \odot \boldsymbol{x}_{2} \neq \boldsymbol{x}_{2} \odot \boldsymbol{x}_{1}$ if $\boldsymbol{x}_{1}$ and $\boldsymbol{x}_{2}$ are distinct elements with the same norm. However, elements with distinct norms do commute. More generally, if $\boldsymbol{x}_{1}, \ldots, \boldsymbol{x}_{n}$ are elements in $\mathrm{E}$ such that exactly one element has maximal norm, $\boldsymbol{x}^{*}$ say, then $\boldsymbol{x}_{1} \odot \cdots \odot \boldsymbol{x}_{n}=\boldsymbol{x}^{*}$ does not depend on the order of the $\boldsymbol{x}_{i}$ 's.

Proposition 3.9. The process $\boldsymbol{X}$ defined by (3.11) admits the Lepage representation

$$
\boldsymbol{X} \stackrel{d}{=} \bigodot_{i=1}^{\infty} \Gamma_{i}^{-1 / \alpha} \boldsymbol{Z}^{(i)}
$$


with $\left\{\Gamma_{i}, i \geq 1\right\}$ and $\left\{\boldsymbol{Z}^{(i)}, i \geq 1\right\}$ as in (3.10) and the operation $\odot$ is taken componentwise. Furthermore, the process $\boldsymbol{X}$ is stable with respect to the operation $\odot$ in the sense that

$$
n^{-1 / \alpha} \bigodot_{i=1}^{n} \boldsymbol{X}^{(i)} \stackrel{d}{=} \boldsymbol{X}
$$

for every $n \geq 1, \boldsymbol{X}^{(1)}, \ldots, \boldsymbol{X}^{(n)}$ being independent copies of $\boldsymbol{X}$.

PROOF. The representation (3.12) is simply a rewriting of the definition of the process $\boldsymbol{X}$, that is,

$$
\bigodot_{i=1}^{\infty} \Gamma_{i}^{-1 / \alpha} \boldsymbol{Z}^{(i)}=\mathcal{T}(\Pi)
$$

where $\Pi \sim \operatorname{PPP}(\boldsymbol{v})$. Let $n \geq 1, \Pi_{1}, \ldots, \Pi_{n}$ be i.i.d. copies of $\Pi$ and $\boldsymbol{X}^{(1)}, \ldots, \boldsymbol{X}^{(n)}$ be independent copies of $\boldsymbol{X}$. Since $\mathcal{T}$ is 1 -homogeneous, we have

$$
n^{-1 / \alpha} \bigodot_{i=1}^{n} \boldsymbol{X}^{(i)}=\mathcal{T}\left(n^{-1 / \alpha} \Pi_{1} \cup \cdots \cup n^{-1 / \alpha} \Pi_{n}\right) \stackrel{d}{=} \mathcal{T}(\Pi),
$$

since $n^{-1 / \alpha} \Pi_{1} \cup \cdots \cup n^{-1 / \alpha} \Pi_{n} \sim \operatorname{PPP}(v)$.

3.4. Extremal indices and $m$-dependent approximation. The purpose of this section is to investigate more advanced properties of the process $\boldsymbol{X}$ defined by (3.11) such as existence of extremal indices and $m$-dependent tail equivalent approximations. Anti-clustering is also discussed in the next section. For the sake of generality, we do not restrict our study to the process (3.11) but rather consider a large class of processes constructed on the Poisson particle system $\Pi \sim \operatorname{PPP}(v)$.

Let us first introduce the notion of extremal index that provides an insight in the dependence structure of a stationary regularly varying time series. For a time series $\xi \in \mathrm{RV}_{\alpha}\left([0, \infty)^{\mathbb{Z}},\left(a_{n}\right), \boldsymbol{v}\right)$, we compare the growth rates of

$$
M_{n}=\max _{1 \leq h \leq n} \xi_{h} \quad \text { and } \quad \widetilde{M}_{n}=\max _{1 \leq h \leq n} \widetilde{\xi}_{h},
$$

where the random variables $\widetilde{\xi}_{h}$ are independent copies of $\xi_{0}$. Regular variation and independence imply that $\widetilde{M}_{n} / a_{n}$ converges to a standard $\alpha$-Fréchet distribution, that is,

$$
\lim _{n \rightarrow \infty} \mathbb{P}\left(a_{n}^{-1} \max _{1 \leq h \leq n} \widetilde{\xi}_{h} \leq x\right)=\mathrm{e}^{-x^{-\alpha}}
$$

for all $x>0$. Under assumptions discussed below, one can prove that

$$
\lim _{n \rightarrow \infty} \mathbb{P}\left(a_{n}^{-1} \max _{1 \leq h \leq n} \xi_{h} \leq x\right)=\mathrm{e}^{-\theta x^{-\alpha}},
$$


for $x>0$ where $\theta \in[0,1]$ is called the extremal index. If $\theta=0$, we have $a_{n}^{-1} M_{n} \stackrel{P}{\rightarrow} 0$ : the maximum has a slower growth rate in the dependent case. When $\theta>0$, the maximum grows at rate $a_{n}$ as in the independent case. The extremal index can also be defined as the limit, if it exists,

$$
\theta=\lim _{n \rightarrow \infty} \log \frac{\mathbb{P}\left(\max _{1 \leq h \leq n} \xi_{h} \leq a_{n}\right)}{\mathbb{P}\left(\xi \leq a_{n}\right)^{n}} .
$$

In the abstract framework $X \in \operatorname{RV}\left(\mathrm{E}^{\mathbb{Z}},\left(a_{n}\right), \boldsymbol{v}\right)$, we consider, for any 1-homogeneous continuous $H: \mathrm{E} \rightarrow[0, \infty)$, the extremal index (if it exists) of the nonnegative time series $\left\{H\left(\boldsymbol{X}_{h}\right), h \in \mathbb{Z}\right\}$ :

$$
\theta_{H}=\lim _{n \rightarrow \infty} \log \frac{\mathbb{P}\left(\max _{1 \leq h \leq n} H\left(\boldsymbol{X}_{h}\right) \leq a_{n}\right)}{\mathbb{P}\left(H\left(\boldsymbol{X}_{0}\right) \leq a_{n}\right)^{n}} .
$$

The homogeneity and continuity of $H$ ensure that $H(X) \in \operatorname{RV}\left([0, \infty)^{\mathbb{Z}},\left\{a_{n}\right\}, \boldsymbol{v}\right.$ 。 $H^{-1}$ ), provided $\boldsymbol{v} \circ H^{-1}$ is not the null measure.

There exists a vast literature on the extremal index and several conditions have been introduced that ensure the existence of a positive extremal index. Building on Chernick, Hsing and McCormick (1991) and using the tail measure through the tail process introduced by Basrak and Segers (2009), we will only consider here a condition based on $m$-dependent tail equivalent approximations. An E-valued time series $\boldsymbol{X}$ is called $m$-dependent if the $\sigma$-fields $\sigma\left(\boldsymbol{X}_{h}, h \leq h_{0}\right)$ and $\sigma\left(\boldsymbol{X}_{h}, h \geq\right.$ $\left.h_{0}+m+1\right)$ are independent for all $h_{0} \in \mathbb{Z}$. In particular, a stationary 0-dependent time series is a series of independent and identically distributed random variables.

DEFINITION 3.10. A process $\boldsymbol{X}$ is said to have a tail equivalent approximation if there exists a sequence of processes $\left\{\boldsymbol{X}^{(m)}, m \geq 1\right\}$ such that

$$
\lim _{m \rightarrow \infty} \limsup _{n \rightarrow \infty} n \mathbb{P}\left(\mathrm{d}_{\mathrm{E}}\left(\boldsymbol{X}_{h} / a_{n}, \boldsymbol{X}_{h}^{(m)} / a_{n}\right)>\varepsilon\right)=0 .
$$

The relationship between $m$-dependent tail equivalent approximation and existence of an extremal index is made clear in the following theorem. Since the extremal index is essentially defined for nonnegative time series, we focus on that case.

LEMMA 3.11. Let $\boldsymbol{X}^{(m)} \in \operatorname{RV}\left([0, \infty)^{\mathbb{Z}},\left\{a_{n}\right\}, \boldsymbol{v}^{(m)}\right)$ be stationary and $m$ dependent. Then $\boldsymbol{X}^{(m)}$ has a positive extremal index equal to the maximal index $\theta_{\tau_{0}}^{(m)}$ of $\boldsymbol{v}^{(m)}$ associated to the map $\tau_{0}$ defined on $[0, \infty)^{\mathbb{Z}}$ by $\tau(\boldsymbol{x})=x_{0}$. If moreover $\boldsymbol{X}^{(m)}$ is a tail equivalent approximation of a nonnegative time series $\boldsymbol{X}$ and if the limit $\lim _{m \rightarrow \infty} \theta_{\tau_{0}}^{(m)}$ exists, then it is the extremal index of $\boldsymbol{X}$.

PROOF. Since an $m$-dependent sequence is $\alpha$ mixing with arbitrary fast rate, the existence of the extremal index $\theta$ is proved by Basrak and Segers [(2009), 
Theorem 4.5] and is given by $\theta^{(m)}=\mathbb{P}\left(\max _{i \geq 1} Y_{i}^{(m)} \leq 1\right)$. Thus $\theta=\theta_{\tau_{0}}$ by Remark 2.21. The second statement is a consequence of Chernick, Hsing and McCormick (1991), Proposition 1.4.

Based on this result, we now prove the existence of the extremal index $\theta_{H}$ the process $\boldsymbol{X}$ considered in (3.11). The process $\boldsymbol{X}$ is defined by means of the stationary $\mathcal{N}_{0}(\mathrm{E})$-valued sequence $\boldsymbol{P}=\left\{P_{h}(\Pi), h \in \mathbb{Z}\right\}$ and the map $T$ introduced in the proof of Theorem 3.7 but the specific form of $T$ is irrelevant and only 1homogeneity and continuity are needed. Therefore, we will first prove that the stationary sequence $\boldsymbol{P}$ admits an $m$-dependent tail equivalent approximation and then obtain the extremal index of time series derived from $\boldsymbol{P}$.

THEOREM 3.12. Let $\boldsymbol{v}$ be a tail measure on $\mathrm{E}^{\mathbb{Z}}$ and $\Pi \sim \operatorname{PPP}(\boldsymbol{v})$ be the associated particle process. Consider the stationary $\mathcal{N}_{0}(\mathrm{E})$-valued process $\boldsymbol{P}=$ $\left\{P_{h}(\Pi), h \in \mathbb{Z}\right\}$. If $\boldsymbol{v}$ has a dissipative representation (2.16), then $\boldsymbol{P}$ has an $m$ dependent tail-equivalent approximation.

Proof. Note first that $\Pi$ can be expressed as

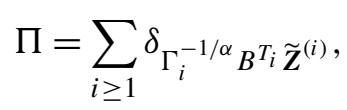

where $\delta_{\Gamma_{i}^{-1 / \alpha}}$ is a Poisson point process on $(0, \infty)$ with mean measure $v_{\alpha}, \widetilde{\boldsymbol{Z}}^{(i)}$ are i.i.d. copies of the process $\widetilde{\boldsymbol{Z}}$ in (2.16), $B$ is the shift operator and $\sum_{i=1}^{\infty} \delta_{T_{i}}$ is a Poisson point process on $\mathbb{Z}$ with mean measure the counting measure on $\mathbb{Z}$, independent of everything else. Indeed, it suffices to check that the mean measure of the point process on the right-hand side of (3.17) is $\boldsymbol{v}$. This follows from (2.16).

We now define the $m$-dependent approximation $\boldsymbol{P}^{(m)}$ of $\boldsymbol{P}$. For $m \geq 1$, define

$$
\boldsymbol{P}_{h}^{(m)}=\sum_{i \geq 1} \delta_{\Gamma_{i}^{-1 / \alpha}} \widetilde{\boldsymbol{Z}}_{h-T_{i}}^{(i)} \mathbb{1}\left\{\left|h-T_{i}\right| \leq m\right\} .
$$

We must now check the tail equivalence condition (3.16). By stationarity, it suffices to check it for $h=0$. That is, we must prove that for all $\varepsilon>0$,

$$
\lim _{m \rightarrow \infty} \limsup _{n \rightarrow \infty} n \mathbb{P}\left(\rho\left(a_{n}^{-1} \boldsymbol{P}_{0}, a_{n}^{-1} \boldsymbol{P}_{0}^{(m)}\right)>\varepsilon\right)=0 .
$$

Set $R_{m}=\bigvee_{i=1}^{\infty} \Gamma_{i}^{-1 / \alpha}\left\|\widetilde{\boldsymbol{Z}}_{-T_{i}}^{(i)}\right\|_{\mathrm{E}} \mathbb{1}\left\{\left|T_{i}\right|>m\right\}$. For $r>a_{n}^{-1} R_{m}, a_{n}^{-1} \boldsymbol{P}_{0}^{(m)}$ and $a_{n}^{-1} \boldsymbol{P}_{0}$ have the same points on $B_{r}^{c}$. Therefore,

$$
\rho\left(a_{n}^{-1} \boldsymbol{P}_{0}, a_{n}^{-1} \boldsymbol{P}_{0}^{(m)}\right)=\int_{0}^{a_{n} R_{m}}\left(\rho_{r}\left(a_{n}^{-1} \boldsymbol{P}_{0}, a_{n}^{-1} \boldsymbol{P}_{0}^{(m)}\right) \wedge 1\right) \mathrm{e}^{-r} \mathrm{~d} r \leq a_{n} R_{m} .
$$

Thus (3.18) will be obtained as a consequence of

$$
\lim _{m \rightarrow \infty} \limsup _{n \rightarrow \infty} n \mathbb{P}\left(R_{m}>a_{n} \varepsilon\right)=0 .
$$


To prove (3.19), note that for nonnegative random variables $Z_{i}, i \geq 1$, since $\Gamma_{1}^{-1 / \alpha}$ has a Fréchet distribution, we have

$$
\begin{aligned}
\mathbb{P}\left(\bigvee_{i=1}^{\infty} \Gamma_{i}^{-1 / \alpha} Z_{i}>x\right) & \leq \sum_{i=1}^{\infty} \mathbb{P}\left(\Gamma_{i}^{-1 / \alpha} Z_{i}>x\right) \\
& \leq \sum_{i=1}^{\infty} \mathbb{P}\left(\Gamma_{1}^{-1 / \alpha} Z_{i}>x\right)=\sum_{i=1}^{\infty}\left(1-\mathrm{e}^{-x^{-\alpha} \mathbb{E}\left[Z_{i}^{\alpha}\right]}\right)
\end{aligned}
$$

Therefore, if $\sum_{i=1}^{\infty} \mathbb{E}\left[Z_{i}^{\alpha}\right]<\infty$, we obtain by dominated convergence

$$
\limsup _{x \rightarrow \infty} x^{\alpha} \mathbb{P}\left(\bigvee_{i=1}^{\infty} \Gamma_{i}^{-1 / \alpha} Z_{i}>x\right) \leq \sum_{i=1}^{\infty} \mathbb{E}\left[Z_{i}^{\alpha}\right]
$$

Applying this bound to $R_{m}$, we obtain by dominated convergence theorem

$$
\lim _{m \rightarrow \infty} \limsup _{n \rightarrow \infty} n \mathbb{P}\left(R_{m}>a_{n} \varepsilon\right) \leq \lim _{m \rightarrow \infty} \sum_{i=1}^{\infty} \mathbb{E}\left[\left\|\boldsymbol{Z}_{0}\right\|_{\mathrm{E}}^{\alpha} \mathbb{1}\left\{\left|T_{i}\right|>m\right\}\right]=0 .
$$

This proves (3.19).

To a function $H: \mathcal{N}_{0}(\mathrm{E}) \rightarrow[0, \infty)$, we associate the function $\widehat{H}: \mathrm{E}^{\mathbb{Z}} \rightarrow$ $[0, \infty)^{\mathbb{Z}}$ defined by $\widehat{H}(\boldsymbol{x})=\left\{H\left(\delta_{\boldsymbol{x}_{h}}\right), h \in \mathbb{Z}\right\}, \boldsymbol{x} \in \mathrm{E}^{\mathbb{Z}}$.

COROLLARY 3.13. Under the assumptions of Theorem 3.12, let $H: \mathcal{N}_{0}(\mathrm{E}) \rightarrow$ $[0, \infty)$ be a Lipschitz continuous 1-homogeneous function such that $\boldsymbol{v}(\{\hat{H}(\boldsymbol{x})>$ $1\})=1$. Then the time series $\boldsymbol{X}_{H}=\left\{H \circ P_{h}(\Pi), h \in \mathbb{Z}\right\}$ is in $\mathrm{RV}\left([0, \infty)^{\mathbb{Z}},\left\{n^{1 / \alpha}\right\}\right.$, $\left.\boldsymbol{v}_{H}\right)$ with $\boldsymbol{v}_{H}=\boldsymbol{v} \circ \widehat{H}^{-1}$, has an $m$-dependent tail equivalent approximation and an extremal index equal to the maximal index $\theta_{\tau}$ associated to $\boldsymbol{v}$ and the map $\tau$ defined on $\mathrm{E}^{\mathbb{Z}}$ by $\tau(\boldsymbol{x})=H\left(\delta_{\boldsymbol{x}_{0}}\right)$.

ProOF. We will apply Lemma 3.11 and Theorem 3.12. Let $\boldsymbol{P}^{(m)}$ be the $m$ dependent approximation of $\boldsymbol{P}$ defined in the proof of Theorem 3.12. Then the time series $\boldsymbol{X}^{(m)}$ defined by $\boldsymbol{X}_{h}^{(m)}=H \circ P_{h}(\Pi), h \in \mathbb{Z}$ is $m$ dependent and regularly varying by Lemma 3.2. By Lemma 3.11, its extremal index $\theta^{(m)}$ is given by

$$
\theta^{(m)}=\frac{\mathbb{E}\left[\max _{|h| \leq m} \bar{H}^{\alpha}\left(\widetilde{\boldsymbol{Z}}_{h}\right)\right]}{\mathbb{E}\left[\sum_{|h| \leq m} \bar{H}^{\alpha}\left(\widetilde{\boldsymbol{Z}}_{h}\right)\right]} .
$$

The tail equivalence condition (3.16) holds by the Lipschitz property of $H$. Thus the sequence $\left\{\boldsymbol{X}^{(m)}\right\}$ is a tail equivalent approximation of $\boldsymbol{X}$ and we can apply Lemma 3.11 which proves (by application of the dominated convergence theorem) that the extremal index of $\boldsymbol{X}$ is

$$
\theta=\lim _{m \rightarrow \infty} \theta^{(m)}=\frac{\mathbb{E}\left[\max _{h \in \mathbb{Z}} \bar{H}^{\alpha}\left(\widetilde{\boldsymbol{Z}}_{h}\right)\right]}{\mathbb{E}\left[\sum_{h \in \mathbb{Z}} \bar{H}^{\alpha}\left(\widetilde{\boldsymbol{Z}}_{h}\right)\right]} .
$$


3.5. The anti-clustering condition. In the literature of time series and extremal index, the anti-clustering condition introduced by Davis and Hsing (1995) plays quite an important role; see, for example, Janssen et al. (2018), Mikosch and Wintenberger (2016), Basrak and Tafro (2016), Basrak, Krizmanić and Segers (2012). Let us first define the notion of anti-clustering for a stationary regularly varying sequence.

DEFINITION 3.14. A stationary time series $\boldsymbol{X} \in \mathrm{RV}\left(\mathrm{E}^{\mathbb{Z}},\left(a_{n}\right), \boldsymbol{v}\right)$ satisfies the anti-clustering condition if there exists an intermediate sequence $r_{n} \rightarrow \infty, r_{n} / n \rightarrow$ 0 , such that

$$
\lim _{m \rightarrow \infty} \limsup _{n \rightarrow \infty} \mathbb{P}\left(\max _{m \leq|h| \leq r_{n}} \mathrm{~d}_{\mathrm{E}}\left(\boldsymbol{X}_{t} / a_{n}, \mathbf{0}_{\mathrm{E}}\right)>u \mid \mathrm{d}_{\mathrm{E}}\left(\boldsymbol{X}_{0} / a_{n}, \mathbf{0}_{\mathrm{E}}\right)>u\right)=0 .
$$

When $E=\mathbb{R}$, we retrieve the classical anti-clustering condition of Davis and Hsing (1995), Condition 2.8. Although we have not used anti-clustering in our analysis of extremal index (Corollary 3.13), we show below that, for the class of processes considered, anti-clustering is equivalent to the existence of a dissipative representation for $\boldsymbol{v}$. This suggests that assuming the existence of a dissipative representation for $\boldsymbol{v}$ in Corollary 3.13 is not a too strong condition.

THEOREM 3.15. The following statements are equivalent:

(i) $v$ has a dissipative representation (2.16);

(ii) the process $\left(P_{h}(\Pi)\right)_{h \in \mathbb{Z}}$ satisfies the anti-clustering condition in $\left(\mathcal{N}_{0}(\mathrm{E})\right)^{\mathbb{Z}}$ in $[0, \infty)^{\mathbb{Z}}$;

(iii) for all $H$ as in Corollary 3.13, $\boldsymbol{X}_{H}$ satisfies the anti-clustering condition in $[0, \infty)^{\mathbb{Z}}$;

(iv) the max-stable process $\left\{\left\|P_{h}(\Pi)\right\|_{\mathrm{E}}, h \in \mathbb{Z}\right\}$ satisfies the anti-clustering condition $[0, \infty)^{\mathbb{Z}}$.

PRoOf. Since the process $\left\{\left\|P_{h}(\Pi)\right\|_{\mathrm{E}}, h \in \mathbb{Z}\right\}$ is max-stable, the equivalence between (i) and (iv) is proved in Debicki and Hashorva (2016), Theorem 2.1. The implication (ii) $\Rightarrow$ (iii) is a consequence of the Lipshitz property of $H$; the implication (iii) $\Rightarrow$ (iv) is trivial since the map $\|\cdot\|_{E}$ satisfies the condition of Corollary 3.13. Conversely, (iv) implies (ii) since $\frac{1}{2}\left(\left\|P_{h}(\Pi)\right\|_{\mathrm{E}} \wedge 1\right) \leq$ $\mathrm{d}_{\mathcal{N}_{0}(\mathrm{E})}\left(a_{n}^{-1} P_{h}(\Pi), \mathbf{0}_{\mathcal{M}}\right) \leq\left\|P_{h}(\Pi)\right\|$ E by (3.3) and (3.4).

\section{APPENDIX A: CONVERGENCE IN $\mathcal{M}_{0}\left(\mathcal{N}_{0}(\mathrm{~F})\right)$}

For $\mu \in \mathcal{M}_{0}\left(\mathcal{N}_{0}(\mathrm{~F})\right)$, we denote by $\mathcal{B}_{\mu}$ the set of Borel sets $B \subset \mathcal{N}_{0}(\mathrm{~F})$ that are bounded away from zero and such that $\mu(\pi(\partial B)>0)=0$, with $\partial B$ the boundary of $B$. 
THEOREM A.1. Let $\mu, \mu_{1}, \mu_{2}, \ldots \in \mathcal{M}_{0}\left(\mathcal{N}_{0}(\mathrm{~F})\right)$. The following statements are equivalent:

(i) $\mu_{n} \stackrel{\mathcal{M}_{0}\left(\mathcal{N}_{0}(\mathrm{~F})\right)}{\longrightarrow} \mu$ as $n \rightarrow \infty$.

(ii) $\mu_{n} \stackrel{\text { fidi }}{\longrightarrow} \mu$ as $n \rightarrow \infty$, in the sense that

$$
\mu_{n}\left(\pi\left(A_{i}\right)=m_{i}, 1 \leq i \leq k\right) \rightarrow \mu\left(\pi\left(A_{i}\right)=m_{i}, 1 \leq i \leq k\right) \quad \text { as } n \rightarrow \infty
$$

for all $k \geq 1,\left(m_{1}, \ldots, m_{k}\right) \in \mathbb{N}^{k} \backslash\{0\}$ and $A_{1}, \ldots, A_{k} \in \mathcal{B}_{\mu}$.

(iii) for all bounded continuous $f: \mathrm{F} \rightarrow[0, \infty)$ vanishing on a neighborhood of $\mathbf{0}_{\mathrm{F}}$,

$$
\int_{\mathcal{N}_{0}(\mathrm{~F})}\left(1-\mathrm{e}^{-\pi(f)}\right) \mu_{n}(\mathrm{~d} \pi) \longrightarrow \int_{\mathcal{N}_{0}(\mathrm{~F})}\left(1-\mathrm{e}^{-\pi(f)}\right) \mu(\mathrm{d} \pi) \quad \text { as } n \rightarrow \infty
$$

with $\pi(f)=\int_{\mathrm{F}} f(x) \pi(\mathrm{d} x)$.

This theorem is similar to the characterization of weak convergence of probability measure on $\mathcal{N}_{0}(F)$ in terms of their finite dimensional distributions and Laplace functional by Zhao (2016), Theorem 3.10 and Corollary 3.11. We consider here $M_{0}$-convergence of measures with possibly infinite total mass, so that we exclude in (ii) the event $\left\{\pi\left(A_{i}\right)=0,1 \leq i \leq k\right\}$ that may have infinite mass and we use in (iii) a modified Laplace transform with $1-\mathrm{e}^{-\pi(f)}$ instead of $\mathrm{e}^{-\pi(f)}$ so as to ensure that the integrals are finite.

PROOF. We begin with some notation and preliminaries that will be used throughout the proofs below. We denote by $B_{\mathrm{F}, r}^{c}\left(\right.$ resp., $\left.B_{\mathcal{N}_{0}(\mathrm{~F}), r}^{c}\right)$ the complement of the ball with center 0 and radius $r>0$ in $F$ [resp., $\left.\mathcal{N}_{0}(F)\right]$. The bounds (3.3) and (3.4), imply that for $r \leq 1$,

$$
B_{\mathcal{N}_{0}(\mathrm{~F}), r}^{c} \subset\left\{\pi\left(B_{\mathrm{F}, r}^{c}\right)>0\right\} \subset B_{\mathcal{N}_{0}(\mathrm{~F}), r / 4}^{c} .
$$

Let $\mu \in \mathcal{M}\left(\mathcal{N}_{0}(\mathrm{~F})\right)$ be fixed and consider a sequence $r_{i} \downarrow 0$ such that $\mu\left(\partial B_{\mathcal{N}_{0}(\mathrm{~F}), r_{i}}^{c}\right)=\mu\left(\pi\left(\partial B_{\mathrm{F}, r_{i}}^{c}\right)>0\right)=0$ for all $i \geq 1$. By Hult and Lindskog [(2006), Theorem 2.2], the $M_{0}$-convergence $\mu_{n} \stackrel{M_{0}}{\longrightarrow} \mu$ is equivalent to the weak convergence $\mu_{n}^{\left(r_{i}\right)} \stackrel{w}{\longrightarrow} \mu^{\left(r_{i}\right)}$ for all $i \geq 1$, where $\mu_{n}^{(r)}$ (resp. $\mu^{(r)}$ ) denotes the restriction of $\mu_{n}$ (resp., $\mu$ ) to $B_{\mathcal{N}_{0}(\mathrm{~F}), r}^{c}$. By the inclusion (A.1), this is also equivalent to the weak convergence $\tilde{\mu}_{n}^{\left(r_{i}\right)} \stackrel{w}{\longrightarrow} \tilde{\mu}^{\left(r_{i}\right)}$ for all $i \geq 1$, where $\tilde{\mu}_{n}^{(r)}$ (resp., $\tilde{\mu}^{(r)}$ ) denotes the restriction of $\mu_{n}$ (resp., $\left.\mu\right)$ to $\left\{\pi\left(B_{\mathrm{F}, r_{i}}^{c}\right)>0\right\}$. The restriction $\tilde{\mu}_{n}^{\left(r_{i}\right)}$ will be useful because they behave well with respect to finite dimensional distributions.

The weak convergence $\mu_{n}^{\left(r_{i}\right)} \stackrel{w}{\longrightarrow} \mu^{\left(r_{i}\right)}$ (or $\tilde{\mu}_{n}^{\left(r_{i}\right)} \stackrel{w}{\longrightarrow} \tilde{\mu}^{\left(r_{i}\right)}$ ) of finite measures can be characterized as in Zhao [(2016), Theorem 3.10 and Corollary 3.11] by the weak convergence of finite dimensional distributions or pointwise convergence of Laplace functionals. Note that the result and proof in Zhao (2016) are given for 
weak convergence of probability measures only, but they are easily extended to finite measures since weak convergence is then equivalent to convergence of the total mass together with weak convergence of the normalized measures.

Proof of $(i) \Rightarrow($ ii $)$. From the preliminary discussion, the $M_{0}$-convergence $\mu_{n} \stackrel{M_{0}}{\longrightarrow} \mu$ implies, for all $i \geq 1$, the convergence of $\mu_{n}\left(B_{\mathcal{N}_{0}(\mathrm{~F}), r_{i}}^{c}\right) \rightarrow \mu\left(B_{\mathcal{N}_{0}(\mathrm{~F}), r_{i}}^{c}\right)$ and the weak convergence of the finite dimensional distributions $\mu_{n}^{\left(r_{i}\right)} \stackrel{f i d i}{\longrightarrow} \mu^{\left(r_{i}\right)}$. This entails the convergence of finite dimensional distributions in the sense of $i i$ ) because any set $A \in \mathcal{B}_{\mu}$ is bounded away from zero and hence in $B_{\mathcal{N}_{0}(\mathrm{~F}), r_{i}}^{c}$ for $r_{i}$ small enough,

Proof of $($ ii $) \Rightarrow$ (iii). It is enough to prove that (ii) implies weak convergence of the finite dimensional distributions $\tilde{\mu}_{n}^{\left(r_{i}\right)} \stackrel{f i d i}{\longrightarrow} \tilde{\mu}^{\left(r_{i}\right)}$ for all $r_{i} \geq 1$. Let $k \geq 1$, $A_{1}, \ldots, A_{k} \in \mathcal{B}_{\mu}$ and $m_{1}, \ldots, m_{k} \geq 0$. Setting $A_{0}=B_{\mathrm{F}, r_{i}}^{c} \in \mathcal{B}_{\mu}$, we have

(A.2) $\tilde{\mu}_{n}^{\left(r_{i}\right)}\left(\pi\left(A_{j}\right)=m_{j}, 1 \leq j \leq k\right)=\tilde{\mu}_{n}\left(\pi\left(A_{0}\right)>0, \pi\left(A_{j}\right)=m_{j}, 1 \leq j \leq k\right)$

and (ii) implies convergence to

(A.3) $\tilde{\mu}^{\left(r_{i}\right)}\left(\pi\left(A_{j}\right)=m_{j}, 1 \leq j \leq k\right)=\tilde{\mu}\left(\pi\left(A_{0}\right)>0, \pi\left(A_{j}\right)=m_{j}, 1 \leq j \leq k\right)$.

This proves $\tilde{\mu}_{n}^{\left(r_{i}\right)} \stackrel{\text { fidi }}{\longrightarrow} \tilde{\mu}^{\left(r_{i}\right)}$ and $\mu_{n} \stackrel{M_{0}}{\longrightarrow} \mu$.

Proof of (iii) $\Rightarrow(i)$. We prove that (iii) implies that, for all $i \geq 1$, the measures $\tilde{\mu}_{n}^{\left(r_{i}\right)}, \tilde{\mu}^{\left(r_{i}\right)}$ have finite total mass and converge weakly $\tilde{\mu}_{n}^{\left(r_{i}\right)} \stackrel{w}{\longrightarrow} \tilde{\mu}^{\left(r_{i}\right)}$ as $n \rightarrow \infty$. We first prove convergence of the total mass

$$
\tilde{\mu}_{n}^{\left(r_{i}\right)}\left(\mathcal{N}_{0}(\mathrm{~F})\right)=\mu_{n}\left(\pi\left(B_{\mathrm{F}, r_{i}}^{c}\right)>0\right) \rightarrow \tilde{\mu}^{\left(r_{i}\right)}\left(\mathcal{N}_{0}(\mathrm{~F})\right)=\mu\left(\pi\left(B_{\mathrm{F}, r_{i}}^{c}\right)>0\right) .
$$

Consider approximating functions $h_{l}^{+}(x) \downarrow \mathbb{1}\left\{x \in \mathrm{cl} B_{\mathrm{F}, r_{i}}^{c}\right\}$ and $h_{l}^{-}(x) \uparrow \mathbb{1}\{x \in$ int $B_{\mathrm{F}, r_{i}}^{c}$ \} that are continuous with values in $[0,1]$ and vanish on a neighborhood of $0_{F}$. The notation $\mathrm{cl}$ and int stands for the closure and interior of the set, respectively.

$$
\begin{aligned}
\int_{\mathcal{N}_{0}(\mathrm{~F})}\left(1-\mathrm{e}^{-t \pi\left(h_{l}^{-}\right)}\right) \mu_{n}(\mathrm{~d} \pi) & \leq \int_{\mathcal{N}_{0}(\mathrm{~F})}\left(1-\mathrm{e}^{-t \pi\left(B_{\mathrm{F}, r_{i}}^{c}\right)}\right) \mu_{n}(\mathrm{~d} \pi) \\
& \leq \int_{\mathcal{N}_{0}(\mathrm{~F})}\left(1-\mathrm{e}^{-t \pi\left(h_{l}^{+}\right)}\right) \mu_{n}(\mathrm{~d} \pi) .
\end{aligned}
$$

The left- and right-hand sides in the previous inequalities converge, and hence are bounded uniformly in $n \geq 1$. Furthermore, since $\pi\left(B_{\mathrm{F}, r_{i}}^{c}\right)$ takes values in $\{0,1,2, \ldots\}$, the quantity

$$
\int_{\mathcal{N}_{0}(\mathrm{~F})}\left(1-\mathrm{e}^{-t \pi\left(B_{\mathrm{F}, r_{i}}^{c}\right)}\right) \mu_{n}(\mathrm{~d} \pi)=\sum_{m \geq 1}\left(1-\mathrm{e}^{-t m}\right) \mu_{n}\left(\pi\left(B_{\mathrm{F}, r_{i}}^{c}\right)=m\right)
$$

satisfies

$$
\left(1-\mathrm{e}^{-t}\right) \mu_{n}\left(\pi\left(B_{\mathrm{F}, r_{i}}^{c}\right)>0\right) \leq \int_{\mathcal{N}_{0}(\mathrm{~F})}\left(1-\mathrm{e}^{-t \pi\left(B_{\mathrm{F}, r_{i}}^{c}\right)}\right) \mu_{n}(\mathrm{~d} \pi) \leq \mu_{n}\left(\pi\left(B_{\mathrm{F}, r_{i}}^{c}\right)>0\right) .
$$


We deduce $\sup _{n \geq 1} \mu_{n}\left(\pi\left(B_{\mathrm{F}, r_{i}}^{c}\right)>0\right)<\infty$ and this holds for all $i \geq 1$. Then, letting $n \rightarrow \infty$ in

$$
\begin{aligned}
\int_{\mathcal{N}_{0}(\mathrm{~F})}\left(1-\mathrm{e}^{-t \pi\left(h_{l}^{-}\right)}\right) \mu_{n}(\mathrm{~d} \pi) & \leq \mu_{n}\left(\pi\left(B_{\mathrm{F}, r_{i}}^{c}\right)>0\right) \\
& \leq\left(1-\mathrm{e}^{-t}\right)^{-1} \int_{\mathcal{N}_{0}(\mathrm{~F})}\left(1-\mathrm{e}^{-t \pi\left(h_{l}^{+}\right)}\right) \mu_{n}(\mathrm{~d} \pi),
\end{aligned}
$$

we get

$$
\begin{aligned}
& \liminf _{n \rightarrow \infty} \mu_{n}\left(\pi\left(B_{\mathrm{F}, r_{i}}^{c}\right)>0\right) \geq \int_{\mathcal{N}_{0}(\mathrm{~F})}\left(1-\mathrm{e}^{-t \pi\left(h_{l}^{-}\right)}\right) \mu(\mathrm{d} \pi), \\
& \limsup _{n \rightarrow \infty} \mu_{n}\left(\pi\left(B_{\mathrm{F}, r_{i}}^{c}\right)>0\right) \leq\left(1-\mathrm{e}^{-t}\right)^{-1} \int_{\mathcal{N}_{0}(\mathrm{~F})}\left(1-\mathrm{e}^{-t \pi\left(h_{l}^{+}\right)}\right) \mu(\mathrm{d} \pi) .
\end{aligned}
$$

Letting $l \rightarrow \infty$ and $t \rightarrow \infty$, monotone convergence entails that the right-hand side in the last two inequalities converge to $\mu\left(\pi\left(\mathrm{cl} B_{\mathrm{F}, r_{i}}^{c}\right)>0\right)$ and $\mu\left(\pi\left(\right.\right.$ int $\left.B_{\mathrm{F}, r_{i}}^{c}\right)>$ $0)$, respectively. These two quantities are equal because we have chose $r_{i}$ such that $B_{\mathrm{F}, r_{i}}^{c} \in \mathcal{B}_{\mu}$, that is, $\mu\left(\pi\left(\partial B_{\mathrm{F}, r_{i}}^{c}\right)>0\right)=0$. Consequently, $\mu_{n}\left(\pi\left(B_{\mathrm{F}, r_{i}}^{c}\right)>0\right) \rightarrow$ $\mu\left(\pi\left(B_{\mathrm{F}, r_{i}}^{c}\right)>0\right)$ as $n \rightarrow \infty$, proving (A.4).

To prove that $\tilde{\mu}_{n}^{\left(r_{i}\right)} \stackrel{w}{\longrightarrow} \tilde{\mu}^{\left(r_{i}\right)}$ using the Laplace functional, it is enough to prove

$$
\lim _{n \rightarrow \infty} \int_{\mathcal{N}_{0}(\mathrm{~F})} \mathrm{e}^{-\pi(f)} \tilde{\mu}_{n}^{\left(r_{i}\right)}(\mathrm{d} \pi)=\int_{\mathcal{N}_{0}(\mathrm{~F})} \mathrm{e}^{-\pi(f)} \tilde{\mu}\left(r_{i}\right)(\mathrm{d} \pi)
$$

for all bounded continuous $f: \mathrm{F} \rightarrow[0, \infty)$ vanishing on a neighborhood of $\mathbf{0}_{\mathrm{F}}$. In view of equation (A.4), this is equivalent to

$$
\lim _{n \rightarrow \infty} \int_{\mathcal{N}_{0}(\mathrm{~F})}\left(1-\mathrm{e}^{-\pi(f)}\right) \tilde{\mu}_{n}^{\left(r_{i}\right)}(\mathrm{d} \pi)=\int_{\mathcal{N}_{0}(\mathrm{~F})}\left(1-\mathrm{e}^{-\pi(f)}\right) \tilde{\mu}\left(r_{i}\right)(\mathrm{d} \pi) .
$$

The proof is similar to that of Equation (A.4) where the measures $\mu_{n}(\mathrm{~d} \pi)$ and $\mu(\mathrm{d} \pi)$ are replaced throughout the proof by $\left(1-\mathrm{e}^{-\pi(f)}\right) \mu_{n}(\mathrm{~d} \pi)$ and $(1-$ $\left.\mathrm{e}^{-\pi(f)}\right) \mu(\mathrm{d} \pi)$, respectively. Details are left to the reader for the sake of brevity. It is useful to note that

$$
\left(1-\mathrm{e}^{-t \pi\left(h_{l}^{ \pm}\right)}\right)\left(1-\mathrm{e}^{-\pi(f)}\right)=\left(1-\mathrm{e}^{-\pi\left(t h_{l}^{ \pm}\right)}\right)+\left(1-\mathrm{e}^{-\pi(f)}\right)-\left(1-\mathrm{e}^{-\pi\left(f+t h_{l}^{ \pm}\right)}\right)
$$

so that (iii) allows to deal with the limit of the integrals as $n \rightarrow \infty$.

\section{APPENDIX B: LEMMAS FOR THE PROOF OF THEOREM 3.7}

The Prohorov distance $\varrho_{\mathrm{E}}$ between two bounded measures $\mu, v$ on a Borel space $E$ is defined by Daley and Vere-Jones [(2003), Section A2.5]

$$
\begin{aligned}
\varrho \mathrm{E}(\mu, \nu)= & \inf \left\{\varepsilon \geq 0: \mu(A) \leq \nu\left(A^{\varepsilon}\right)+\varepsilon,\right. \\
& \left.\nu(A) \leq \mu\left(A^{\varepsilon}\right)+\varepsilon \text { for all closed sets } A\right\}
\end{aligned}
$$


LEMMA B.1. Let $\mu, v$ be two point measures on a metric space $(\mathrm{E}, d)$. Then $\varrho_{\mathrm{E}}(\mu, v) \geq|\mu(\mathrm{E})-v(\mathrm{E})|$. If $\boldsymbol{x}, \boldsymbol{y} \in \mathrm{E}$, then $\varrho_{\mathrm{E}}\left(\delta_{\boldsymbol{x}}, \delta_{\boldsymbol{y}}\right) \leq d(\boldsymbol{x}, \boldsymbol{y}) \wedge 1$.

Proof. Assume for instance that $\mu(\mathrm{E})=n$ and $v(\mathrm{E})=k$ with $k<n$. Let $\boldsymbol{x}_{1}, \ldots, \boldsymbol{x}_{n}$ be the points of $\mu$ and let $A=\left\{\boldsymbol{x}_{1}, \ldots, \boldsymbol{x}_{n}\right\}$. Then $A$ is closed, $\mu(A)=$ $n$ and for all $\varepsilon>0, v\left(A^{\varepsilon}\right) \leq k$. This proves that $\varrho_{\mathrm{E}}(\mu, v) \geq n-k$. The second statement is in Dudley (2002), Section 11.3, page 394.

Recall from Section 3.3.1 the definition of the set $\mathcal{N}_{0}^{\sharp}(E)$ and the map $T$ and the definition of the metric $\rho$ in (3.2).

LEMMA B.2. The subset $\mathcal{N}_{0}^{\sharp}(\mathrm{E})$ is open in $\mathcal{N}_{0}(\mathrm{E})$ and the map $T: \mathcal{N}_{0}(\mathrm{E}) \rightarrow \mathrm{E}$ is continuous on $\mathcal{N}_{0}^{\sharp}(\mathrm{E})$.

Proof. Let $\pi \in \mathcal{N}_{0}^{\sharp}(\mathrm{E})$ and $m=\|\pi\|_{\mathrm{E}}$. Then there exists $\eta>0$ such that $\pi$ has exactly one point in $B(T(\pi), \eta)$. A point measure $\pi^{\prime} \in \mathcal{N}_{0}(\mathrm{E})$ has either zero point or 1 or at least two points in $B_{m-\eta}^{c}$. By Lemma B.1, in the first and last cases, $\rho_{r}\left(\pi, \pi^{\prime}\right) \wedge 1=1$, hence

$$
\rho\left(\pi, \pi^{\prime}\right) \geq \int_{m-\eta}^{\infty} \mathrm{e}^{-r} \mathrm{~d} r \geq \mathrm{e}^{-m}
$$

Thus, if $\rho\left(\pi, \pi^{\prime}\right)<\mathrm{e}^{-m}$, then $\pi^{\prime}$ has exactly one point in $B_{m-\eta}^{c}$, which is therefore its single largest point and $\pi^{\prime} \in \mathcal{N}_{0}^{\sharp}(E)$. This proves that $\mathcal{N}_{0}^{\sharp}(E)$ is open. By Lemma B.1 again, for $r>m-\eta$, we have $\rho_{r}\left(\pi, \pi^{\prime}\right) \wedge 1=\mathrm{d}_{\mathrm{E}}\left(T(\pi), T\left(\pi^{\prime}\right)\right) \wedge 1$ thus

$$
\rho\left(\pi, \pi^{\prime}\right) \geq \int_{m-\eta}^{\infty} \mathrm{e}^{-r}\left(\mathrm{~d}_{\mathrm{E}}\left(T(\pi), T\left(\pi^{\prime}\right)\right) \wedge 1\right) \mathrm{d} r \geq\left(\mathrm{d}_{\mathrm{E}}\left(T(\pi), T\left(\pi^{\prime}\right)\right) \wedge 1\right) \mathrm{e}^{-m} .
$$

This proves that $T$ is continuous at $\pi$.

LEMMA B.3. Let $\left(\mathrm{S}, \mathrm{d}_{\mathrm{S}}\right)$ be a metric space and $g: \mathrm{S} \rightarrow \mathrm{F}=\mathrm{E}^{\mathbb{Z}}$. Then $S$ is continuous with respect to the distance $\mathrm{d}_{\mathrm{F}}$ defined in (3.7) if and only if $g_{j}: \mathrm{S} \rightarrow \mathrm{E}$ defined by $g_{j}(s)=(g(s))_{j}$ for $s \in \mathrm{S}$ is continuous for all $j \in \mathbb{Z}$.

ProOf. The direct implication is trivial. We prove the converse. Assume that $g_{j}$ is continuous for all $j$. Fix $s_{0} \in \mathrm{S}, \varepsilon \in(0,1)$ and choose $K$ such that $2^{-K} \leq \varepsilon / 4$. By assumption, there exists $\eta$ (which depends on $\varepsilon$ and $K$ ) such that for all $j \in$ $\{-K, \ldots, K\}$ and $s \in \mathrm{S}$ such $\mathrm{d}_{\mathrm{S}}\left(\boldsymbol{s}_{0}, \boldsymbol{s}\right) \leq \eta, \mathrm{d}_{\mathrm{E}}\left(g_{j}\left(\boldsymbol{s}_{0}\right), g_{j}(\boldsymbol{s})\right) \leq \varepsilon / 2$. This yields

$$
\begin{aligned}
\mathrm{d}_{\mathrm{F}}\left(g\left(s_{0}\right), g(s)\right) & =\sum_{j \in \mathbb{Z}} 2^{-|j|} \mathrm{d}_{\mathrm{E}}\left(g_{j}\left(s_{0}\right), g_{j}(s)\right) \wedge 1 \\
& \leq \frac{\varepsilon}{6} \sum_{|j| \leq K} 2^{-|j|}+\sum_{|j|>K} 2^{-|j|} \leq \varepsilon .
\end{aligned}
$$


Acknowledgements. Two anonymous referees and the Associate Editors are acknowledged for various suggestions that have improved the paper; in particular, the introduction of the Hopf decomposition in Section 2.5 was suggested by a referee.

\section{REFERENCES}

Aaronson, J. (1997). An Introduction to Infinite Ergodic Theory. Mathematical Surveys and Monographs 50. Amer. Math. Soc., Providence, RI. MR1450400

Basrak, B., Krizmanić, D. and Segers, J. (2012). A functional limit theorem for dependent sequences with infinite variance stable limits. Ann. Probab. 40 2008-2033. MR3025708

BASRAK, B. and Planinić, H. (2018). A note on vague convergence of measures. Available at arXiv:1803.07024.

BASRAK, B. and SEgERS, J. (2009). Regularly varying multivariate time series. Stochastic Process. Appl. 119 1055-1080. MR2508565

BASRAK, B. and TAFRO, A. (2016). A complete convergence theorem for stationary regularly varying multivariate time series. Extremes 19 549-560. MR3535966

Buhl, S. and KLÜPPElBERG, C. (2016). Anisotropic Brown-Resnick space-time processes: Estimation and model assessment. Extremes $19627-660$. MR3558348

Chernick, M. R., Hsing, T. and MCCORMiCK, W. P. (1991). Calculating the extremal index for a class of stationary sequences. Adv. in Appl. Probab. 23 835-850. MR1133731

DAley, D. J. and Vere-Jones, D. (2003). An Introduction to the Theory of Point Processes. Vol. I: Elementary Theory and Methods, 2nd ed. Springer, New York. MR1950431

DAVIS, R. A. and Hsing, T. (1995). Point process and partial sum convergence for weakly dependent random variables with infinite variance. Ann. Probab. 23 879-917. MR1334176

Davis, R. A., KlÜppelberg, C. and Steinkohl, C. (2013). Statistical inference for max-stable processes in space and time. J. R. Stat. Soc. Ser. B. Stat. Methodol. 75 791-819. MR3124792

DE HAAN, L. (1984). A spectral representation for max-stable processes. Ann. Probab. 121194 1204. MR0757776

DEBICKI, K. and HASHORVA, E. (2016). Extremal indices of max-stable stationary processes. Unpublished manuscript.

Dieker, A. B. and Mikosch, T. (2015). Exact simulation of Brown-Resnick random fields at a finite number of locations. Extremes 18 301-314. MR3351818

Dombry, C. and KABLuCHKo, Z. (2017). Ergodic decompositions of stationary max-stable processes in terms of their spectral functions. Stochastic Process. Appl. 127 1763-1784. MR3646430

Dudley, R. M. (2002). Real Analysis and Probability. Cambridge Studies in Advanced Mathematics 74. Cambridge Univ. Press, Cambridge. Revised reprint of the 1989 original. MR1932358

HASHORVA, E. (2018). Representations of max-stable processes via exponential tilting. Stochastic Process. Appl. 128 2952-2978. MR3834849

Hult, H. and Lindskog, F. (2006). Regular variation for measures on metric spaces. Publ. Inst. Math. (Beograd) (N.S.) 80 121-140. MR2281910

JANSSEN, A. (2017). Spectral tail processes and max-stable approximations of multivariate regularly varying time series. Available at arXiv:1704.06179.

Janssen, A., Mikosch, T., Rezapour, M. and Xie, X. (2018). The eigenvalues of the sample covariance matrix of a multivariate heavy-tailed stochastic volatility model. Bernoulli $241351-$ 1393. MR3706796

Kallenberg, O. (2017). Random Measures, Theory and Applications. Probability Theory and Stochastic Modelling 77. Springer, Cham. MR3642325

KuliK, R. (2016). Editorial: Special issue on time series extremes. Extremes 19 463-466. MR3535962 
LINDSKOG, F., RESNICK, S. I. and RoY, J. (2014). Regularly varying measures on metric spaces: Hidden regular variation and hidden jumps. Probab. Surv. 11 270-314. MR3271332

Mikosch, T. and Wintenberger, O. (2016). A large deviations approach to limit theory for heavy-tailed time series. Probab. Theory Related Fields 166 233-269. MR3547739

Owada, T. and SAmorodnitsky, G. (2012). Tail measures of stochastic processes or random fields with regularly varying tails. Preprint.

PlAninić, H. and SouliER, P. (2018). The tail process revisited. Extremes. DOI:10.1007/s10687018-0312-1.

Segers, J., Zhao, Y. and Meinguet, T. (2017). Polar decomposition of regularly varying time series in star-shaped metric spaces. Extremes 20 539-566. MR3679981

SMITH, R. L. and WeISSMANNN (1996). Characterization and estimation of the multivariate extremal index. Preprint. Available on http://www.stat.unc.edu/postscript/rs/extremal.pdf.

Zнао, Y. (2016). Point processes in a metric space. ArXiv e-prints.

C. DOMBRY

LABORATOIRE DE MATHÉMATIQUES DE BESANÇON

UNIVERSITÉ BOURGOGNE

FRANCHE-COMTÉ

UMR CNRS 6623

16 ROUTE DE GRAY

25030 BESANÇON CEDEX

FRANCE

E-MAIL: clement.dombry@univ-fcomte.fr

\author{
E. HASHORVA \\ DÉPARTEMENT DE SCIENCES \\ ACTUARIELLES \\ UNIVERSITÉ DE LAUSANNE \\ QUARTIER UNIL-CHAMBERONNE \\ BÂTIMENT EXTRANEF \\ 1015 LAUSANNE \\ SWITZERLAND \\ E-MAIL: enkelejd.hashorva@unil.ch
}

\author{
P. SOULIER \\ DÉPARTEMEnt de Mathématique \\ ET INFORMATIQUE \\ UNIVERSITÉ PARIS NANTERRE \\ LABORATOIRE MODAL'X \\ 92000 NANTERRE \\ FRANCE \\ E-MAIL: philippe.soulier@u-paris10.fr
}

